\title{
A model for the Z-track phenomenon in GX 5-1 and observational evidence for the physical origins of the $\mathrm{kHz}$ QPO
}

\author{
N. K. Jackson ${ }^{1}$, M. J. Church ${ }^{1,2}$, and M. Bałucińska-Church ${ }^{1,2}$ \\ 1 School of Physics and Astronomy, University of Birmingham, Birmingham, B15 2TT, UK \\ e-mail: njackson@star.sr.bham.ac.uk \\ 2 Astronomical Observatory, Jagiellonian University, ul. Orla 171, 30-244 Cracow, Poland \\ Received 4 August 2008 / Accepted 2 December 2008
}

ABSTRACT

\begin{abstract}
We present the results of a combined investigation of the spectral and kHz QPO evolution around the Z-track in GX 5-1 based on high-quality Rossi-XTE data. In spectral analysis, we find that the Extended ADC emission model provides very good fits to all of the spectra, and the results point clearly to a model for the nature of the Z-track in this source, in agreement with previous results for the similar source GX 340+0. In this model, at the soft apex of the Z-track, the mass accretion rate $\dot{M}$ is at its minimum and the neutron star has its lowest temperature; but as the source moves along the normal branch, the luminosity of the Comptonized emission increases, indicating that $\dot{M}$ increases and the neutron star gets hotter. The measured flux $f$ of the neutron star emission increases by a factor of ten becoming super-Eddington, and we propose that this causes disruption of the inner disk and the formation of jets. In flaring, the luminosity of the dominant Comptonized emission from the accretion disk corona is constant, while the neutron star emission increases, and we propose for the first time that flaring consists of unstable nuclear burning on the neutron star, supported by the agreement between the measured mass accretion rate per unit area $\dot{m}$ at the onset of flaring and the theoretical critical value at which burning becomes unstable. There is a striking correlation between the frequencies of the $\mathrm{kHz} \mathrm{QPO}$ and the ratio of the flux to the Eddington value: $f / f_{\text {Edd }}$, suggesting an explanation of the higher frequency QPO and of its variation along the Z-track. It is well known that a Keplerian orbit in the disk at this frequency corresponds to a position some distance from the neutron star; we propose that the oscillation always occurs at the inner disk edge, which moves radially outwards on the upper normal and horizontal branches as the measured increasing radiation pressure increasingly disrupts the inner disk.
\end{abstract}

Key words. accretion, accretion disks - stars: binaries: close - stars: neutron - X-rays: binaries - X-rays: individuals: GX 5-1

\section{Introduction}

The Z-track sources form the brightest group of low mass X-ray binaries (LMXB) containing a neutron star, with luminosities at or above the Eddington limit. They are characterised by having three distinct branches in a hardness-intensity diagram: the horizontal branch (HB), the normal branch (NB) and the flaring branch (FB) (Hasinger \& van der Klis 1989) showing that major physical changes take place within the sources but the nature of these has not been understood. It has been widely thought that the physical and spectral changes are driven by change of a single parameter along the Z-track, presumably the mass accretion rate (Priedhorsky et al. 1986), assumed to increase monotonically in the direction HB - NB - FB. However, as discussed below, the evidence for this is rather limited. Moreover the variation of the X-ray intensity does not obviously support this as the intensity does not increase monotonically in the direction $\mathrm{HB}-\mathrm{NB}-\mathrm{FB}$, but decreases on the normal branch. Arguably the most important feature of the Z-track sources is the detection of radio emission showing jets to be present but only in the upper normal and horizontal branches (Penninx 1989). The presence of jets was dramatically demonstrated by extended radio observations of the Z-track source Sco X-1 (Fomalont et al. 2001) which revealed radio condensations moving away from the source with velocity $v / c$ of 0.45 . Thus the Z-track sources uniquely provide the possibility of determining the physical conditions within the sources on the part of the Z-track where radio is detected so telling us the conditions needed for the launching of jets. Apart from this an understanding of the Z-track sources is essential to the basic understanding of LMXB in general. Extensive work has been carried out on the timing properties of Z-track sources (van der Klis et al. 1987; Hasinger \& van der Klis 1989) and revealed the existence of quasi-periodic oscillations (QPO) which change along the Z-track. However, analysis of the timing properties has not provided an explanation of the Z-track phenomenon. Spectral analysis is more likely to reveal the nature of the physical changes taking place as directly showing changes in the emission components during the spectral evolution along the Z-track, but spectral studies of the sources have been hindered by lack of agreement over the emission model to be used.

\subsection{Spectral studies of the Z-track sources}

The nature of X-ray emission in LMXB has been controversial essentially because two radically different types of model for the continuum are capable of describing the spectra in which a dominant Comptonized component and also a thermal component are clearly present. Analysis of the dipping class of LMXB led to the "Extended ADC" model in which the dominant component is Comptonized emission from the accretion disk corona (ADC) plus thermal emission from the neutron star (Church \& Bałucińska-Church 1995, 2004). Both spectral and timing analysis showed the Comptonizing ADC to be a region extending over a substantial fraction of the inner accretion disk (typically $15 \%)$ and thin $(H / r \ll 1)$ as discussed in more detail below. The other model is the Eastern model comprising multicolour blackbody emission from the inner accretion disk plus 
Comptonized emission from a central region close to the neutron star (Mitsuda et al. 1989). Previous spectral fitting of the Z-track sources has mostly been based on use of the Eastern model as summarized below. However, there is now a body of evidence that the Comptonizing region is extended (Sect. 1.2) and is not a small central region. Moreover, when the Eastern model was applied to the Z-track sources, it was difficult to interpret the variation of spectral parameters and a physical explanation was often not given.

An extensive investigation of spectral evolution along the Z-track in several sources using Exosat data was carried out by Schulz et al. (1989) and by Schulz and Wijers (1993) using a model with neutron star blackbody emission plus Comptonization. Hasinger et al. (1990) fitted Ginga data on Cyg X-2 with both Western and Eastern models. In the Western model the main change was an increase of blackbody temperature on climbing the normal branch. In the Eastern model, there was a systematic change in the Comptonization $y$-parameter on the normal branch. Because of the ability of both models to fit, it was not thought possible to distinguish between physical models. Consideration of multi-wavelength data and of the timing properties of the source led the authors to conclude that the mass accretion rate $\dot{M}$ increased monotonically in the direction $\mathrm{HB}-\mathrm{NB}-\mathrm{FB}$ although X-ray intensity decreased on the NB. A possible explanation was that a thickening of the inner disk obscured the X-ray emission. The conclusion about $\dot{M}$ led Hasinger et al., following Hasinger (1988) and Lamb (1989), to propose a scenario in which $\dot{M}$ was small on the HB so that a thin disk existed together with a magnetosphere in which radio emission was generated. Increasing $\dot{M}$ resulted in the magnetosphere being enveloped by a vertically expanding disk at the hard apex, suppressing radio emission. At the soft apex $\dot{M}$ exceeds the Eddington value so that strong radiation pressure led to mass ejection above and below the disk. Asai et al. (1994) fitted Ginga data on the NB and FB spectra of GX 5-1 with both Western and Eastern models, but using the Eastern model in its original form consisting of disk blackbody plus blackbody from the neutron star. This work concentrated on the emission feature found at $10 \mathrm{keV}$.

Spectral fitting of Cyg X-2 was carried out by Done et al. (2002) using the Eastern model assuming that the seed photons for Comptonization come from the neutron star or inner disk and so should be described by a blackbody spectrum. It was also assumed that the central Comptonizing cloud would illuminate the disk so that reflection should be present (although in the Z-track sources the height of the inner disk is much greater than that of the neutron star). The spectral fitting indicated an increasing flux of the disk blackbody in the lower normal and flaring branches where it was assumed that $\dot{M}$ increased; however, the disk temperature appeared constant so it was argued that the inner disk radius must increase. The Comptonized flux also remained constant when it might be expected to increase and it was not clear what caused the spectral parameters to vary in the way found. Agrawal \& Sreekumar (2003) used the Eastern model to fit Rossi-XTE spectra of GX $349+2$ in the NB and FB using the form DISKBB + COMPTT, i.e. disk blackbody plus Comptonization with the seed photons having a Wien distribution. The inner disk temperature first increased on the NB then decreased in flaring; the inner disk radius first decreased then increased, suggesting that the inner disk edge was moving to larger radial positions, and the authors proposed that there was thus an outflow of material. The electron temperature of the Comptonizing plasma first increased on the NB then decreased in flaring while the optical depth of the region first decreased then increased. It was suggested that the initial heating caused a density decrease and reduction in optical depth; on the FB the outflow proposed added density to the hot central corona causing the increase of optical depth on this branch.

Spectral fitting of broadband BeppoSAX data was carried out for GX 17+2, GX 349+2 and Cyg X-2 (di Salvo et al. 2000, 2001, 2002). In the case of Cyg X-2, the Eastern model was applied together with a power law to represent a high energy tail, and it was argued that all three sources could be fitted by this model. In Cyg X-2 spectral evolution consisted of an increase of disk blackbody temperature and decrease of blackbody radius moving from the $\mathrm{HB}$ to the NB, suggesting that the inner disk radius was shrinking although an explanation of this was not proposed. There were changes in temperature of the Comptonizing plasma and on the normal branch, the inferred radius of this region fell to $2-3 \mathrm{~km}$, incompatible with the neutron star being the source of seed photons unless there was a marked change in geometry from the normally assumed spherical Comptonizing cloud of the Eastern model. D'Ai et al. (2007) investigated the Z-track in Sco X-1 using extensive numbers of observations with $R X T E$. The Eastern model in the form used by di Salvo et al. was used. The disk blackbody temperature was highest on the flaring branch as expected on the standard assumption that $\dot{M}$ is largest here. The electron temperature of the Comptonizing plasma increased around the $\mathrm{Z}$ having its highest values in flaring, while at the same time the optical depth decreased; however, the reasons for these changes were not clear.

It is difficult to find consistency in the spectral fitting results and with the difficulty of interpreting the evolution of spectral parameters along the Z-track it is fair to say that there is no general concensus on the nature of the Z-track. Much effort was devoted to producing a theoretical model of of accretion in the inner disk in LMXB which proposed detailed physical changes taking place along the Z-track (Lamb 1989; Miller 1990; Psaltis et al. 1995). This involves a magnetosphere at the inner accretion disk, and changes in the geometry and extent of the magnetosphere. The model assumes the main element of the Eastern model that is, a small central Comptonizing region. In the present work we take the approach that the evidence favours an extended Comptonizing region and we test the hypothesis that the Extended ADC model may provide a physically reasonable and consistent explanation of the Z-track source GX 5-1.

\subsection{The extended $A D C$}

Our work on the dipping class of LMXB previously led to our proposal of the extended ADC model comprising blackbody emission from the neutron star plus Comptonized emission from an extended, thin ADC existing as a hot layer above the accretion disk. In the dipping sources with inclination angles between $65^{\circ}$ and $85^{\circ}$, reductions in X-ray intensity take place at the orbital period generally thought to involve absorption in the bulge in the outer disk where the accretion flow impacts. Spectral evolution cannot be described in terms of absorption of a single emission component, but is more complex strongly constraining allowable models. It provides direct evidence for the extended nature of the Comptonized emission as this spectral component is removed only slowly during dipping showing the emitter to be extended. Secondly, the ADC size can be measured directly by the technique of dip ingress timing since the ingress time depends on the size of the major emission component: the Comptonized emission. This has shown that the radial extent of the ADC is typically $50000 \mathrm{~km}$ (Church \& Bałucińska-Church 2004) also showing that the ADC, like the disk is in general thin, with 
height $H$ at radial position $r$ having $H / r \ll 1$. The extended ADC model was shown to give very good fits to the complex spectral evolution in dipping in many observations of the dipping LMXB (Church et al. 1997, 1998a,b, 2005; Bałucińska-Church et al. 1999, 2000; Smale et al. 2001; Barnard et al. 2001). In addition, it was shown able to fit the spectra of all types of LMXB in a survey of LMXB made with ASCA (Church \& BałucińskaChurch 2001) and so was able to fit sources of all inclination angles.

The evidence favouring the extended ADC is thus strong and it is difficult to avoid the conclusion that the main assumption of the Eastern model is invalid.

The main difference between the spectral forms of the extended ADC model and the Eastern model relates to the seed photons. In the Eastern model it is usually assumed that these originate on the neutron star and can be described by a single temperature blackbody or by the Wien approximation. In the extended ADC model the major source of seed photons must be the thermal emission of the disk below the ADC. Because of the temperature gradient $T(r)$ in the disk, the outer portions of the ADC will be above relatively cool parts of the disk providing a large population of soft seed photons ( $k T \sim 0.1 \mathrm{keV}$ or less) and the seed photon spectrum is very different from a blackbody at $\sim 1 \mathrm{keV}$. Comptonization is well represented by a power law extending to energies lower than $0.1 \mathrm{keV}$, with a cut-off at high energies related to the maximum electron energy (see Church \& Bałucińska-Church 2004, for a full discussion). The use of a simple blackbody for the neutron star emission in applying the extended ADC model reflects the general lack of evidence for modification of the blackbody in the atmosphere of the neutron star (Church et al. 2002). Taking into account many spectral studies of LMXB, including the spectra of X-ray bursts, the only evidence for modification is in a minority of bursts, with no evidence in non-burst emission. Church et al. showed that whether modification occurs depends critically on the electron density in the neutron star atmosphere and that this is poorly known.

\subsection{A model for the Z-track}

We previously applied the extended ADC model to high quality Rossi-X-ray Timing Explorer data from the Z-track source GX 340+0 (Church et al. 2006), and found that it fitted the spectra well at all positions on the Z-track, and also clearly suggested a physical model for the phenomenon. In this model, the soft apex between NB and FB is a state of the source in which the intensity and the mass accretion rate were low. Moving onto the NB, there was a large increase in X-ray intensity (count $\mathrm{s}^{-1}$ ) and an increase in the total broadband luminosity by about a factor of two caused by an increase in the luminosity $L_{\mathrm{ADC}}$ of the Comptonized emission of the ADC. It was argued that this increase indicated that $\dot{M}$ was increasing on the NB and was supported by a marked increase in the blackbody temperature of the neutron star from $\sim 1 \mathrm{keV}$ to $\sim 2 \mathrm{keV}$ and even higher values on the HB. This heating of the neutron star means a large increase in $T^{4}$ and so the radiation pressure became very high. There was a simultaneous decrease in emitting area on the neutron star. In this situation, it is appropriate to compare the flux emitted per unit area of the star with the Eddington value (see Sect. 4.1), and the flux increased from a low value till on the HB, it was three times super-Eddington. It was argued that this would disrupt the inner disk deflecting the accretion flow into the vertical direction leading to jet formation, and explaining why jets are detected on the upper NB and HB where the radiation pressure was high. In flaring it was found that $L_{\mathrm{ADC}}$ was constant implying that $\dot{M}$ was constant although the neutron star luminosity increased. The mass accretion rate per unit area of the neutron star closely agreed with the theoretical value for the onset of unstable nuclear burning, and it was proposed thus flaring was due to this.

In the present work, we apply the extended ADC model to high-quality Rossi-XTE observations of the Z-track source GX 5-1 to test the hypothesis that the same physical explanation can explain a second Z-track source. Spectral and timing studies were made using the same selections of data allowing the evolution of $\mathrm{kHz}$ QPO frequency to be directly correlated with the spectral evolution. This revealed a striking dependence of $\mathrm{kHz}$ QPO frequency on the neutron star radiation pressure. In the next section $\mathrm{kHz}$ QPO are briefly reviewed.

\subsection{The $\mathrm{kHz}$ QPO}

Quasi periodic oscillations have been the subject of intense study for many years, and a major discovery of the Rossi X-ray Timing Explorer was that of the twin kilohertz QPO in LMXB. Predictions of millisecond variability pre-date this considerably and Sunyaev (1973) argued that hot clumps of material orbiting in the inner disk around a black hole or neutron star would lead to quasiperiodic variability on millisecond timescales. Kilohertz QPO have now been detected in many LMXB (see reviews of van der Klis 2000, 2006), most of these displaying two peaks at frequencies such as at 600 and $900 \mathrm{~Hz}$, and in the Z-track sources both frequencies increase during movement along the HB towards the hard apex. Rather similar QPO have also been seen in a number of black hole binaries.

In almost all QPO models the higher frequency peak is associated with the orbital frequency at some distance into the accretion disk away from the neutron star; for example, a frequency of $900 \mathrm{~Hz}$ corresponds to a radial position $r$ of about $18 \mathrm{~km}$. In the sonic point beat frequency model (Miller et al. 1998) radiation drag reduces the angular momentum in the disk within a few stellar radii of the neutron star leading to a sonic point where the radial velocity becomes high (Lamb 1989; Miller 1991; Miller $\&$ Lamb 1993, 1996). The upper frequency $v_{2}$ is associated with inhomogeneities orbiting at the sonic point in the inner disk, which are irradiated by X-rays from hot regions rotating on the neutron star leading to a beating of the upper frequency with the spin frequency $v_{\text {spin }}$ so producing the lower frequency QPO. Although earlier observations found the frequency difference $\Delta v$ constant within errors consistent with the model, improved data showed that $\Delta v$ varied around the Z-track. The detection of twin $\mathrm{kHz}$ QPO and X-ray burst oscillations in the accreting millisecond pulsar SAX J1808.4-3658 (Wijnands et al. 2003) led to the conclusion that $\Delta v \sim v_{\text {spin }}$ in some sources, but $v_{\text {spin }} / 2$ in other sources, inconsistent with the model, leading Lamb \& Miller (2003) to propose a relativistic disk-spin resonance model.

The relativistic precession model (Stella \& Vietri 1998) identifies $v_{2}$ with motion of inhomogeneities at the inner disk edge and the lower frequency $v_{1}$ with precession of the inhomogeneities. The disk resonance model (Abramowicz \& Kluźniak 2001) recognizes the preference of the $\mathrm{kHz}$ QPO to have a ratio of 3:2 in their frequencies suggesting a non-linear resonance between normal modes of the disk. This model predicted that twin peaks should also exist in black hole binaries, and the discovery of a second peak in GRO J1655-40 (Strohmayer 2001) was a success of the model. Comparison with data (Abramowicz et al. 2003; Kluźniak et al. 2007) for both neutron star and black hole systems supports the model: black hole systems accurately follow the 3:2 ratio; LMXB show some scatter but a histogram of 
the ratio peaks strongly at 3:2. However, Belloni et al. (2005) have argued that in the case of Sco X-1, the peak in the distribution at a ratio of 1.5 was only marginally significant.

\subsection{The assumed monotonic variation of mass accretion rate}

The assumption of $\dot{M}$ increasing in the direction $\mathrm{HB}-\mathrm{NB}-\mathrm{FB}$ generally, but not universally made, is not supported by our previous results for GX $340+0$, and so we briefly review evidence for and against this.

The evidence for this was based originally on a multiwavelength campaign on Cyg X-2 (Hasinger et al. 1990) involving X-rays, ultraviolet, optical and radio data. Results from IUE (Vrtilek et al. 1990) had increasing intensity for Z-track movement $\mathrm{HB}-\mathrm{NB}-\mathrm{FB}$ implying an increase of $\dot{M}$; however, strong variability interpreted as flaring may have been due to X-ray dipping in which case there is no strong correlation of UV with track position.

The properties of most power spectral features correlate well with position on the Z-track; for example, the frequencies of the HBO (horizontal branch oscillations), i.e. low frequency QPO, increase around the Z-track in the direction $\mathrm{HB}-\mathrm{NB}$ from $\sim 5$ to $\sim 60 \mathrm{~Hz}$ (e.g. Homan et al. 2002). Similarly, NBO (normal branch oscillations) are seen on the NB and in two sources, Sco X-1 and GX 17+2, oscillations are seen in the FB, but only close to the soft apex, and there is a continuous increase of QPO frequency from lower NB to FB (Casella et al. 2006), i.e. in crossing the soft apex between these branches. Similarly, the frequencies of kilohertz QPO increase along the HB where they are observed (e.g. van der Klis 2000). It is general practice to display QPO properties as a function of arc distance $S$ measured along the Z-track in the direction $\mathrm{HB}-\mathrm{NB}-\mathrm{FB}$, and this shows that QPO properties are generally well-correlated with $S$ (e.g. Dieters \& van der Klis 2000) implying a dependence on a single parameter, i.e. $\dot{M}$. It is also generally though that the soft apex represents the position at which $\dot{M}$ increases to the Eddington value (Lamb 1989). In addition, the frequencies of $\mathrm{HBO}$ and $\mathrm{kHz}$ QPO in many models are directly related to $\dot{M}$ (see Homan et al. 2002).

However, in recent years, some authors are beginning to doubt the general assumption of a monotonic increase in $\dot{M}$. Some results conflict with the assumption: e.g. in GX 17+2, the frequency of the HBO first increases, but then decreases on the normal branch (Wijnands et al. 1996; Homan et al. 2002). In ScoX-1 in the neighbourhood of the soft apex there is a rapid increase of QPO frequency (Casella et al. 2000; Dieters \& van der Klis 2000) with $S$ which is inconsistent with the assumption that $S$ is a measure of $\dot{M}$ as the change is over a very short part of the Z-track. Moreover the approximate constancy of NBO frequency in general over most of the NB does not indicate a dependency on $\dot{M}$. Also, in GX $17+2$, the properties of the X-ray bursts sometimes observed do not depend on Z-track position (Kuulkers et al. 2002).

In our previous work on GX 340+0 (Church et al. 2006) we proposed that $\dot{M}$ increased between the soft apex and the hard apex, while on the FB, $\dot{M}$ was constant. The X-ray intensity, the measured broadband luminosity of the source and the luminosity of the Comptonized emission all increased substantially on the NB strongly suggesting that $\dot{M}$ is not decreasing. The argument is often made that the X-ray intensity cannot be a good indicator of $S$ and thus of $\dot{M}$ (e.g. Dieters \& van der Klis 2000) because the intensity increases on both the NB and FB, moving away from the soft apex. However, our work on GX $340+0$ shows this not to be a secure argument, since the intensity increases on the NB because $\dot{M}$ increases, while on the FB, $\dot{M}$ is constant, but strong nuclear burning on the neutron star causes the intensity increase. One aim of the present work is to test whether similar results are found in GX 5-1.

\section{6. $G \times 5-1$}

GX 5-1 is the second brightest persistent LMXB (after Sco X-1), discovered in 1968 (Fisher et al. 1968; Bradt et al. 1968) as a bright X-ray source in the Sagittarius region and classified as a Z-track source by Hasinger \& van der Klis (1989). A threeyear study of the source was made using Ginga (van der Klis et al. 1991) which revealed an extended horizontal branch and an upcurving at the end suggesting a change of behaviour, effectively a fourth branch (Lewin et al. 1992). Analysis of Exosat and Ginga data revealed flaring branch data for the first time (Kuulkers et al. 1994) and a detailed investigation of correlated spectral and timing behaviour was made. It was the first Z-track source found to have QPO (van der Klis et al. 1985) and kilohertz QPO were detected by Wijnands et al. (1998). Radio emission has been detected from GX 5-1, e.g. by Penninx (1989) implying the existence of jets, and it is known to be strongest on the horizontal branch (Berendsen et al. 2000), as for other Z-track sources. Analysis of 1987 Ginga data (Asai et al. 1994) and ASCA data (Asai et al. 2000) showed there was no evidence for an iron $\mathrm{K}$ line, but a Gaussian feature existed at $10 \mathrm{keV}$. To date no satisfactory explanation exists for this line.

\section{Observations and analysis}

GX 5-1 has been observed several times with RXTE. We examined the data in the HEASARC archive remotely producing hardness-intensity plots and selected data which spanned the full Z-track in a relatively short time. It is important not to use data in which sideways movement of the Z-track takes place, broadening the track or even giving several shifted Z-tracks, as we are interested in the changes taking place around a single Z-track. Similarly data separated by months should not be co-added on the assumption that the source was in the same state because portions of the Z-track approximately overlap. Thus a single observation including all branches is preferred, and in view of the very high count rates in the RXTE instruments, there is no need to add further data as the count statistics are already excellent. We thus selected the observation of 1998 November 21-22 having the best coverage of the Z-track in a single observation, consisting of four sub-observations spanning $90 \mathrm{ksec}$.

Data from both the Proportional Counter Array (PCA, Jahoda et al. 1996) and the high-energy X-ray timing experiment (HEXTE) were used. The PCA was in Standard 2 mode with $16 \mathrm{~s}$ resolution and examination of the housekeeping data revealed that all five of the xenon proportional counter units were on during the observation. Standard screening criteria based on the housekeeping data were applied to select data having an offset between the source and telescope pointing of less than $0.02^{\circ}$ and elevation above the Earth's limb greater than $10^{\circ}$. Data were extracted from the top layer of the detector using both left and right anodes. Analysis was carried out using the FTOOLS 5.3.1 package. Lightcurves were generated in the band $1.9-18.5 \mathrm{keV}$ and background files for each PCA data file produced using the facility PCABACKEST, applying the latest "bright" background model for Epoch 3 of the mission (1996 April 15-1999 March 22) which includes the present observation. Deadtime correction 


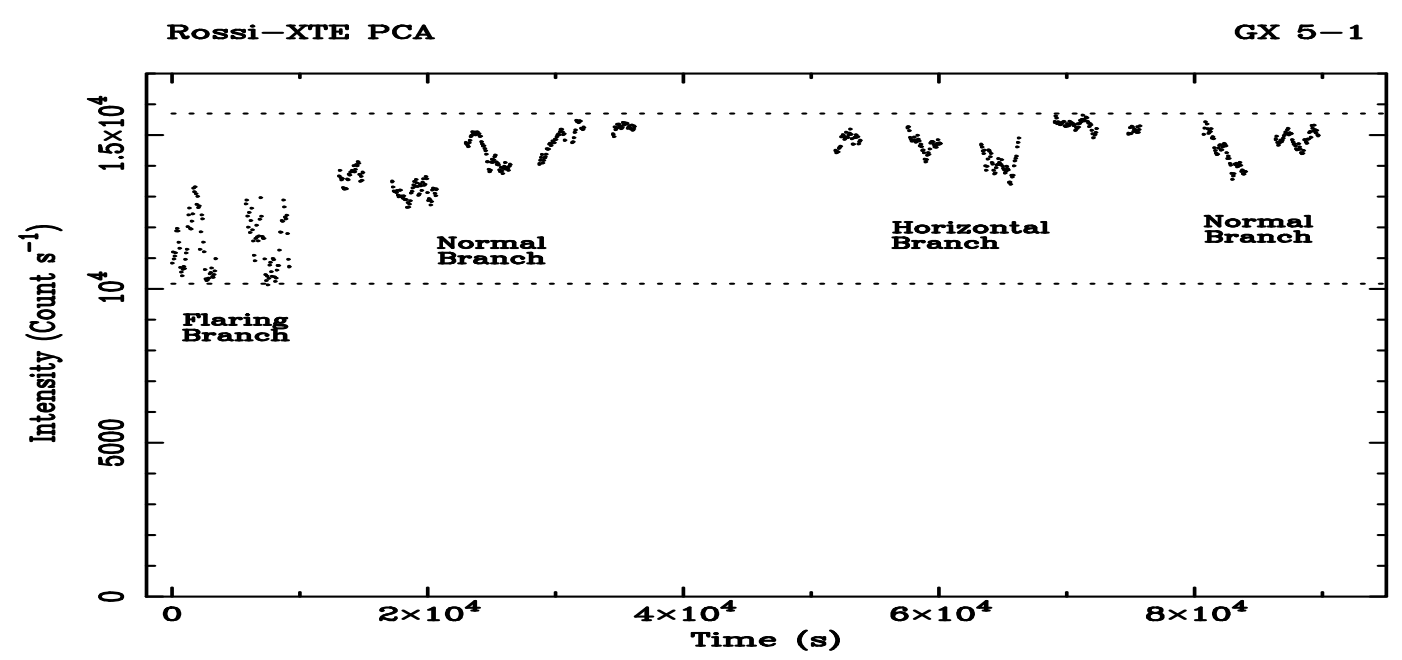

Fig. 1. Background-subtracted deadtime-corrected PCA lightcurve of the November 1998 observation of GX 5-1 with 64 s binning.

Rossi-XTE PCA

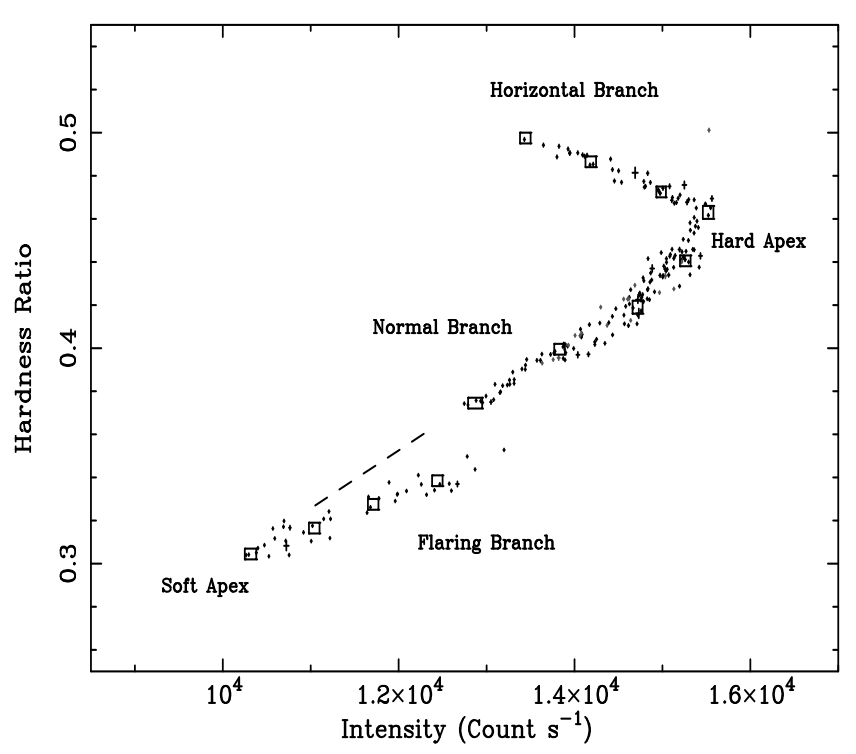

Fig. 2. Z-track of the observation derived from background-subtracted and deadtime-corrected lightcurves with $176 \mathrm{~s}$ binning. The boxes show the ranges of intensity and hardness ratio used for the selection of PCA and HEXTE spectra, and timing data.

was carried out on both source and background files prior to subtraction. Light curves were also made in sub-bands of the above range, and a hardness ratio defined as the ratio of the intensity in the band 7.3-18.5 keV to that in the 4.1-7.3 keV band. Tests were made on suitable binning for the lightcurves and Z-track. In order to reduce scatter in the plot of hardness versus intensity and produce a relatively narrow, well-defined Z-track, it was found that a binning of $176 \mathrm{~s}$ was optimum. In Fig. 1 we show the background-subtracted, deadtime-corrected lightcurve in the band $1.9-18.5 \mathrm{keV}$ with the binning reduced from 176 to $64 \mathrm{~s}$ to allow more detail to be seen. In Fig. 2 we show the backgroundsubtracted, deadtime corrected Z-track with $176 \mathrm{~s}$ binning. We identified each subsection of the lightcurve with its position on the Z-track by making a hardness-intensity plot for each and finding where this lay on the overall Z-track. Each of the 14 subsections was selected using the FTOOL MAKETIME. It can be seen that the intensity of the source varied between two well-defined limits corresponding to the Soft Apex and the Hard Apex of the Z-track as discussed below.
HEXTE data were also extracted as lightcurves and spectra from Cluster 1 of the HEXTE instrument using the FTOOL HXTLCURVE which also provided background files. Deadtime correction was applied using the deadtime coefficients file of February 2000. This allowed simultaneous fitting of PCA and HEXTE spectra, the wider energy band resulting in spectral fitting being better constrained that with the PCA alone.

\section{Results}

From Fig. 1 the strong variability of GX 5-1 during these observations can be seen, and the lightcurve is labelled to show the identifications with the branches of the Z-track. At the start of the observation there is strong flaring with individual flares lasting several thousand seconds. The source then moves to the normal branch with the intensity increasing. Following movement along the normal branch to the hard apex, the source reaches the horizontal branch, the intensity then falling on this branch to intermediate values.

Figure 2 shows the corresponding Z-track having full coverage of the $\mathrm{Z}$ except for the lower part of the normal branch, as the source was in this position during a data gap between subobservations. However, this gap was not a problem as it can be seen from the results in Figs. 4-6 that there is no ambiguity in the behaviour of the source in this part of the Z-track. In the present work, we carry out both spectral fitting and timing analysis for data selected at a sequence of positions along the Z-track. This approach thus allows direct comparison of the QPO results with spectral fitting results for exactly the same data.

Our previous work has suggested the importance of selecting data lying on a smooth curve along the centre of the Z-track (Barnard et al. 2003) so that spectral changes reflect changes along the track and not perpendicular to it. PCA spectra were generated about equally-spaced along the Z-track selecting data within boxes in hardness-intensity by use of a good time interval (GTI) file for each selection. The boxes were 100 count s$^{-1}$ wide in intensity and with a range of hardness ratio of 0.005 . It was checked that the selections used were correct by overlaying the selected data on the full Z-track. In Fig. 2 it can be seen that the boxes are small in comparison with the Z-track as a whole so spectral fitting results relate accurately to a small part of the Z-track. It would clearly be inappropriate to use large boxes extending along a significant fraction of the Z-track as appreciable spectral changes will take place within the box. However, although the boxes used in the present work are small, the count 
Table 1. Counts accumulated in the PCA and HEXTE for each selection; the selections are numbered 1 to 11 starting from the $\mathrm{HB}$ end of the Z-track.

\begin{tabular}{lrr}
\hline \hline Selection & PCA Count & HEXTE Count \\
\hline 1 & $2.04 \times 10^{6}$ & $2.98 \times 10^{3}$ \\
2 & $4.30 \times 10^{6}$ & $6.38 \times 10^{3}$ \\
3 & $10.23 \times 10^{6}$ & $11.69 \times 10^{3}$ \\
4 & $4.69 \times 10^{6}$ & $6.66 \times 10^{3}$ \\
5 & $15.71 \times 10^{6}$ & $15.16 \times 10^{3}$ \\
6 & $2.17 \times 10^{6}$ & $3.81 \times 10^{3}$ \\
7 & $9.78 \times 10^{6}$ & $10.30 \times 10^{3}$ \\
8 & $6.78 \times 10^{6}$ & $6.17 \times 10^{3}$ \\
9 & $3.05 \times 10^{6}$ & $4.52 \times 10^{3}$ \\
10 & $1.63 \times 10^{6}$ & $2.08 \times 10^{3}$ \\
11 & $1.74 \times 10^{6}$ & $1.89 \times 10^{3}$ \\
\hline
\end{tabular}

rates in the PCA and HEXTE are so large that the total count accumulated in each box is very high as shown in Table 1 allowing high quality spectral fitting. Accurate determination of QPO frequencies was also possible, this requiring good count statistics as the fractional modulation is only a few percent.

After initial tests, it was decided to use 11 spectra consisting of three spectra on the horizontal branch, one at the hard apex, four on the normal branch, one at the soft apex, and two in flaring, numbered as spectrum 1 (end of $\mathrm{HB}$ ) to 11 (FB). An extra spectrum close to the peak of flaring (shown in Fig. 2) was found to have insufficient counts for good spectral fitting. Background spectra were generated for each selection. Both source and background data were deadtime corrected using a local facility PCADEAD. Pulse pileup correction has negligible effect on the spectra and so was not made. It was not necessary to regroup spectral channels to a specified minimum count to allow use of the $\chi^{2}$ statistic as the count in all channels was already high. A systematic error of $1 \%$ was added to each channel as usual for PCA data.

HEXTE spectra and background files were produced for each selection using the same GTI files and deadtime-corrected using the FTOOL HXTLCURV. The auxiliary response file (arf) of May 2000, and the response matrix file (rmf) of March 1997 were used in spectral analysis. The rmf file was rebinned to match the actual number of HEXTE channels using RDDESCR and RBNRMF. For both the PCA and HEXTE, the source + background spectra were individually compared with background spectra and spectral fitting only carried out up to the energy at which these became equal, typically $30 \mathrm{keV}$ in the PCA and $40 \mathrm{keV}$ in HEXTE.

\subsection{Spectral analysis}

The motivation of this work is to test the hypothesis that the extended ADC model (Sect. 1) can provide good fits to the spectra of the Z-track sources, and to test the physical model previously proposed to explain the Z-track source GX 340+0 (Church et al. 2006) in the case of GX 5-1. Consequently, the extended ADC model was used in Xspec spectral fitting software in the form BB + CPL as discussed in Sect. 1.

Preliminary fitting showed that the model provided good values of $\chi^{2} /$ d.o.f for all spectra. The residuals exhibited no evidence for an iron line; however, at the peak of flaring, there was evidence for the $10 \mathrm{keV}$ emission feature seen by Asai et al. (1994). This was seen clearly in the spectrum eventually discarded because of insufficient counts, but was also detectable in spectra 10 and 11 on the flaring branch at a significance of about $2 \sigma$. When added to the fitting, the line had little effect on the results, for example, changing the neutron star blackbody radius by about $5 \%$. Thus the model finally used in fitting all of the spectra omitted the line.

In the Z-track sources, it is well-known that the Comptonized emission has a relatively low cut-off energy of a few $\mathrm{keV}$ whereas lower luminosity sources have a higher cut-off energy which can approach $100 \mathrm{keV}$. Broadband data such as from BeppoSAX or Suzaku allow measurement of the cut-off energy and the power law photon index $\Gamma$. But in the Z-track sources including GX 5-1, the low cut-off energy restricts the energy range available for determination of $\Gamma$ and we have taken the approach previously adopted (e.g. Church et al. 2006) of fixing $\Gamma$ at 1.7 , a physically reasonable value (Shapiro et al. 1976). This procedure gave good quality spectral fits, and when a solution was obtained, it was found that $\Gamma$ could be freed, but then remained close to 1.7. Extensive testing also revealed that the results obtained, i.e. the pattern of variation of parameters along the Z-track did not depend on the value of power law index.

The lower energy limit of the PCA, usually set in spectral fitting at $\sim 3 \mathrm{keV}$, does not allow very accurate determination of the column density, and it is often the case that $N_{\mathrm{H}}$ has to be fixed at the Galactic value. In the present case, the Galactic value of $\sim 0.94 \times 10^{22}$ atom $\mathrm{cm}^{-2}$ (Dickey \& Lockman 1990) was tried, but the fits were unacceptable with, for example, $\chi^{2} /$ d.o.f of 288/59 for spectrum 1. Christian \& Swank (1997) also found a value higher than Galactic $\left(2.54 \times 10^{22}\right.$ atom cm $\left.^{-2}\right)$. In the present case, with free $N_{\mathrm{H}}$, the values varied between 5.7 and $6.8 \times 10^{22}$ atom $\mathrm{cm}^{-2}$ along the Z-track and $\chi^{2} /$ d.o.f. became acceptable. Moreover, it was apparent that $N_{\mathrm{H}}$ changed systematically around the Z-track showing not only is the extra absorption intrinsic, but providing evidence for mass release or outflow within the system associated with the physical changes taking place. The same effect was seen in the case of RXTE observations of the Z-track source GX 340+0 (Church et al. 2006). The best-fit to the spectrum of the hard apex is shown in Fig. 3 as both the folded data (with residuals) and as unfolded data. Final fitting results are shown in Table 1 and in the following we discuss the behaviour of the blackbody and Comptonized emission.

Firstly, we show in Fig. 4 the variation of the blackbody temperature $k T_{\mathrm{BB}}$ and blackbody radius $R_{\mathrm{BB}}$ around the Z-track as a function of the total 1-30 keV luminosity $L_{\text {Tot }}$ for which a source distance of $9 \mathrm{kpc}$ (Christian \& Swank 1997) was assumed. The blackbody radius is defined by $L_{\mathrm{BB}}=4 \pi R_{\mathrm{BB}}^{2} \sigma T^{4}$ where $L_{\mathrm{BB}}$ is the blackbody luminosity and $\sigma$ is Stefan's constant. The blackbody parameters change systematically along the $\mathrm{Z}, k T_{\mathrm{BB}}$ having its lowest value of $1.3 \mathrm{keV}$ at the soft apex where $R_{\mathrm{BB}}$ is maximum at $11_{-0.7}^{+1.4} \mathrm{~km}$. Moving along the normal branch, $k T_{\mathrm{BB}}$ increases to $1.9 \mathrm{keV}$ at the hard apex, and continues to increase on the horizontal branch, reaching $2.1 \mathrm{keV}$ at the end of the Z-track. At the soft apex, the value of $R_{\mathrm{BB}}$ indicates that the whole neutron star is emitting, but decreases on the normal branch showing that the emission contracts presumably to an equatorial belt. In Sect. 4.1 we discuss this measured decrease in emitting area on the neutron star showing that it is consistent with the effects of increased radiation pressure. Thus at the soft apex the source appears to be in an undisturbed state with the neutron star emitting from its whole surface at its lowest temperature; we see below that the evidence indicates the mass accretion rate is minimum at this position.

In Fig. 5 we show the variation of the luminosities of the individual emission components $L_{\mathrm{BB}}$ and $L_{\mathrm{ADC}}$ : the neutron star blackbody and the Comptonized emission of the ADC. Firstly, it 
Table 2. Spectral fitting results: $90 \%$ confidence errors are shown.

\begin{tabular}{lrrrrrrr}
\hline \hline Spectrum & $N_{\mathrm{H}}$ & $\begin{array}{r}k T \\
\mathrm{keV}\end{array}$ & Norm $\mathrm{BB}$ & $\begin{array}{c}R_{\mathrm{BB}} \\
\mathrm{km}\end{array}$ & $\begin{array}{c}E_{\mathrm{CO}} \\
\mathrm{keV}\end{array}$ & Norm $_{\mathrm{CPL}}$ & $\chi^{2} /$ d.o.f. \\
\hline Horizontal branch & & & & & & & \\
1 & $6.1 \pm 0.5$ & $2.08 \pm 0.10$ & $7.6 \pm 0.7$ & $5.1 \pm 0.5$ & $5.9 \pm 0.3$ & $19.5 \pm 1.4$ & $32 / 58$ \\
2 & $6.3 \pm 0.5$ & $1.98 \pm 0.10$ & $7.2 \pm 0.8$ & $5.4 \pm 0.6$ & $5.8 \pm 0.2$ & $21.2 \pm 1.5$ & $24 / 58$ \\
3 & $6.5 \pm 0.4$ & $1.93 \pm 0.08$ & $7.4 \pm 0.8$ & $5.8 \pm 0.5$ & $5.6 \pm 0.2$ & $23.4 \pm 1.5$ & $46 / 58$ \\
Hard apex & & & & & & & \\
4 & $6.8 \pm 0.5$ & $1.87 \pm 0.09$ & $7.2 \pm 0.1$ & $6.07 \pm 0.6$ & $5.6 \pm 0.2$ & $25.3 \pm 1.8$ & $38 / 58$ \\
Normal branch & & & & & & & \\
5 & $6.6 \pm 0.4$ & $1.77 \pm 0.07$ & $6.6 \pm 1.1$ & $6.5 \pm 0.5$ & $5.4 \pm 0.1$ & $25.5 \pm 1.7$ & $37 / 58$ \\
6 & $6.5 \pm 0.5$ & $1.70 \pm 0.10$ & $5.6 \pm 0.6$ & $6.5 \pm 0.8$ & $5.1 \pm 0.1$ & $26.9 \pm 0.8$ & $41 / 58$ \\
7 & $6.4 \pm 0.5$ & $1.54 \pm 0.07$ & $5.0 \pm 0.1$ & $7.5 \pm 0.7$ & $4.9 \pm 0.1$ & $26.1 \pm 2.0$ & $40 / 58$ \\
8 & $6.2 \pm 0.5$ & $1.41 \pm 0.06$ & $4.8 \pm 0.2$ & $8.9 \pm 0.8$ & $4.7 \pm 0.1$ & $24.4 \pm 2.3$ & $38 / 58$ \\
Soft apex & & & & & & & \\
9 & $5.7 \pm 0.7$ & $1.31 \pm 0.05$ & $6.0 \pm 0.2$ & $11.3_{-0.7}^{+1.4}$ & $3.7 \pm 0.1$ & $25.1 \pm 3.3$ & $74 / 58$ \\
Flaring branch & & & & & & & \\
10 & $5.8 \pm 0.7$ & $1.39 \pm 0.06$ & $8.6 \pm 0.2$ & $12.0 \pm 1.0$ & $3.6 \pm 0.2$ & $25.6 \pm 3.8$ & $48 / 58$ \\
11 & $5.9 \pm 0.7$ & $1.46 \pm 0.05$ & $11.6 \pm 0.1$ & $12.7 \pm 0.8$ & $3.5 \pm 0.2$ & $27.2 \pm 4.0$ & $40 / 58$ \\
\hline
\end{tabular}

Column densities are in units of $10^{22}$ atom $\mathrm{cm}^{-2}$; the normalization of the blackbody is in units of $10^{37} \mathrm{erg} \mathrm{s}^{-1}$ for a distance of $10 \mathrm{kpc}$, the normalization of the cut-off power law is in units of photon $\mathrm{cm}^{-2} \mathrm{~s}^{-1} \mathrm{keV}^{-1}$ at $1 \mathrm{keV}$.

RXTE PCA HEXTE

GX 5-1

RXTE PCA HEXTE

GX 5-1
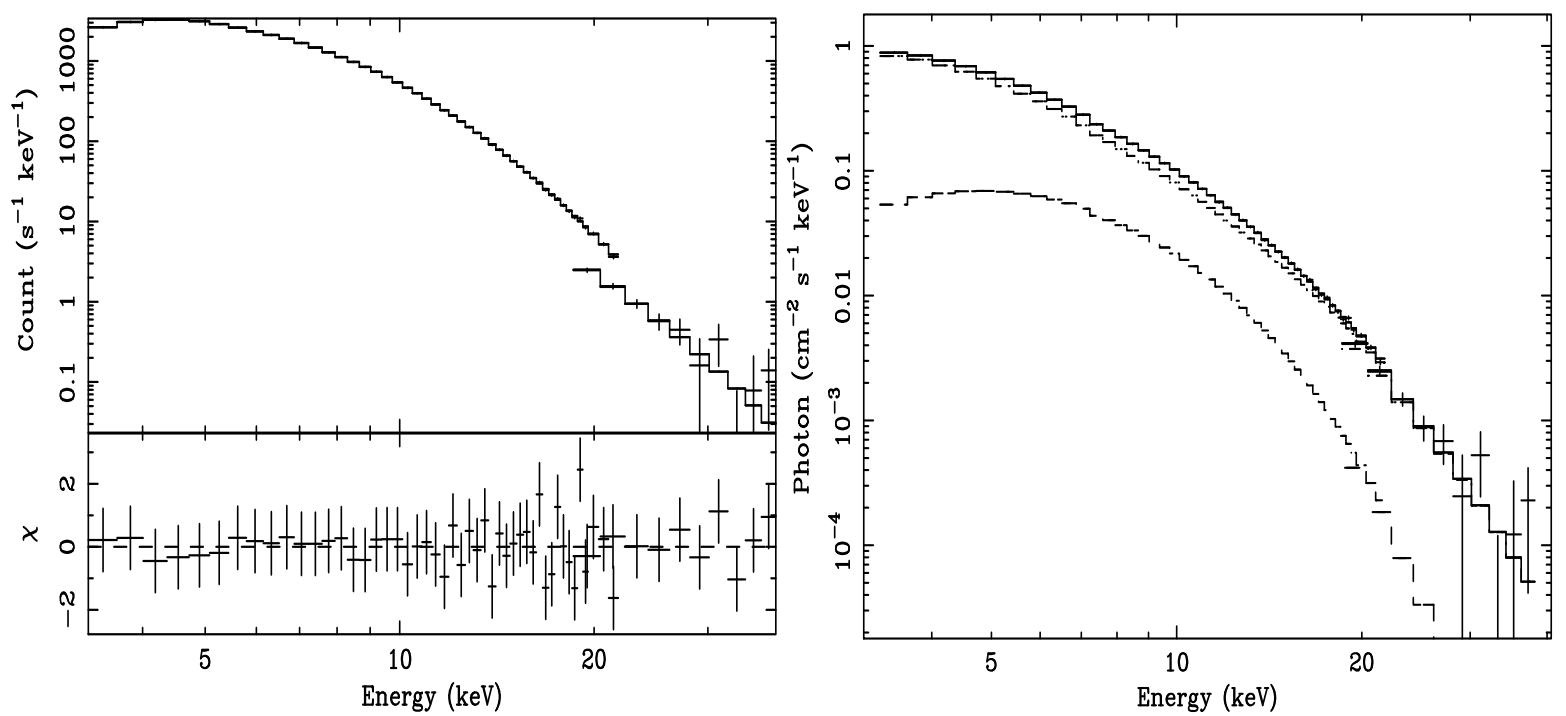

Fig. 3. Left: best-fit to the spectrum of the hard apex with residuals, right: the corresponding unfolded spectrum.

is clear that the Comptonized emission is dominant, its luminosity being ten times larger than that of the blackbody. However, in flaring, the blackbody luminosity increases by a factor of two to $\sim 40 \%$ of the total at the peak of flaring. Moving away from the soft apex on the normal branch there is a strong increase of $L_{\mathrm{ADC}}$ by $40 \%$ on the normal branch, with the PCA intensity increasing from $\sim 10000$ to $\sim 15500$ count $^{-1}$. The increase in X-ray intensity is, of course, unquestionable.

The significance of the ADC luminosity increase on the normal branch was assessed by fitting the variation of $L_{\mathrm{ADC}}$ with $L_{\mathrm{Tot}}$ (lower panel of Fig. 5) also shown in Table 3 using only the normal branch data. A linear fit gave a slope of $0.945 \pm 0.35$, where the errors at $90 \%$ confidence strongly exclude a slope of zero. The probability that the slope is zero (i.e. testing the hypothesis that the luminosity is constant) is less than $0.006 \%$ so that this increase of ADC luminosity on the normal branch is highly significant. Figure 5 shows that the increase of X-ray intensity in climbing the normal branch is due to the increase in
$L_{\mathrm{ADC}}$. We suggest that it is unlikely that $L_{\mathrm{ADC}}$ could increase so substantially without an increase in mass accretion rate $\dot{M}$, and this is also indicated by the increase in neutron star blackbody temperature. In previous work, it was often assumed that the Z-track was produced by the mass accretion rate $\dot{M}$ varying monotonically in the direction HB - NB - FB (Sect. 1), although this conflicted with the known increase of X-ray intensity between the soft and hard apex. We propose here based on the measured definite increase of $L_{\mathrm{ADC}}$ that $\dot{M}$ increases on the normal branch between the soft apex and hard apex, in the opposite direction to that normally assumed. The behaviour of the neutron star blackbody is more complicated consisting of a temperature increase but an area decrease such that the overall luminosity does not change substantially as discussed in Sect. 4.1 and so we should not expect $L_{\mathrm{BB}}$ to follow $\dot{M}$ in a simple way.

The generally accepted idea that $\dot{M}$ increases monotonically around the Z-track explains flaring as a luminosity increase due to high and presumably variable $\dot{M}$. However, it is clear from 
Rossi-XTE $\quad$ PCA + HEXTE

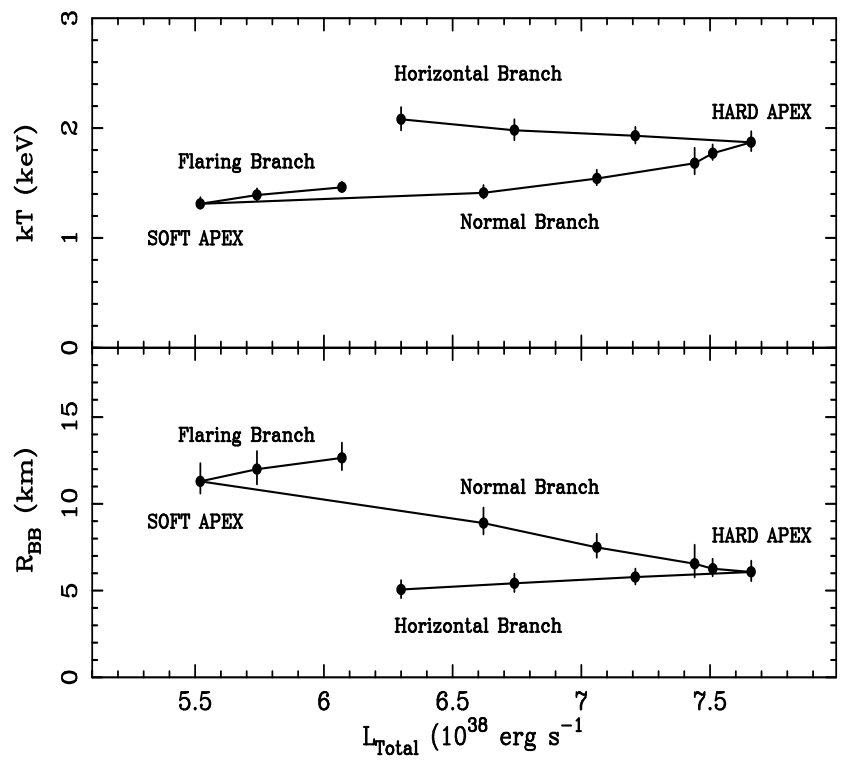

Fig. 4. The neutron star blackbody emission: upper panel: the blackbody temperature; lower panel: the blackbody radius.

Table 3. Total luminosity, blackbody luminosity and ADC luminosity in the band $1-30 \mathrm{keV}$ in units of $\mathrm{erg} \mathrm{s}^{-1}$.

\begin{tabular}{lrrr}
\hline \hline Spectrum & $\begin{array}{r}L_{\mathrm{Tot}} \\
\left(10^{38}\right)\end{array}$ & $\begin{array}{r}L_{\mathrm{BB}} \\
\left(10^{37}\right)\end{array}$ & $\begin{array}{r}L_{\mathrm{ADC}} \\
\left(10^{38}\right)\end{array}$ \\
\hline 1 & 6.30 & $6.19 \pm 0.57$ & $5.68 \pm 0.44$ \\
2 & 6.74 & $5.82 \pm 0.61$ & $6.16 \pm 0.44$ \\
3 & 7.21 & $5.99 \pm 0.65$ & $6.62 \pm 0.41$ \\
4 & 7.67 & $5.82 \pm 0.77$ & $7.08 \pm 0.50$ \\
5 & 7.51 & $4.97 \pm 0.81$ & $6.98 \pm 0.46$ \\
6 & 7.44 & $4.42 \pm 0.81$ & $6.98 \pm 0.60$ \\
7 & 7.06 & $4.08 \pm 1.30$ & $6.66 \pm 0.54$ \\
8 & 6.62 & $3.86 \pm 1.40$ & $6.24 \pm 0.61$ \\
9 & 5.52 & $4.86 \pm 1.40$ & $5.04 \pm 0.68$ \\
10 & 5.74 & $6.94 \pm 1.40$ & $5.06 \pm 0.75$ \\
11 & 6.07 & $9.40 \pm 0.81$ & $5.12 \pm 0.75$ \\
\hline
\end{tabular}

As is normal practice, we do not quote errors in the total luminosity.

Fig. 5 that $L_{\mathrm{ADC}}$ is constant within errors on the FB indicating that $\dot{M}$ does not change in flaring. The blackbody luminosity however, clearly increases and we propose that flaring must consist of luminosity increase due to unstable thermonuclear burning on the surface of the neutron star, a suggestion that has not previously been made. During flaring, there is a relatively small increase of blackbody temperature, but the blackbody radius rises to $\sim 13 \mathrm{~km}$ for spectrum 11 , and would be even larger at the peak of flaring. Values of $R_{\mathrm{BB}}$ substantially larger than the probable neutron star radius have been found previously in GX 5-1 (Christian \& Swank 1997), and a type of radius expansion known in X-ray bursts may be taking place, with the emitting region expanding beyond the normal surface of the neutron star.

Figure 6 shows the variation of the ADC Comptonized emission parameters: the normalization and the cut-off energy $E_{\mathrm{CO}}$. Firstly, there is an increase of normalization moving on the NB away from the soft apex, while $E_{\mathrm{CO}}$ increases, these factors combining to give the observed increase of $L_{\mathrm{ADC}}$ (Fig. 5). Secondly, comparison of Figs. 4 and 6 shows that $E_{\mathrm{CO}}$ is well correlated with $k T_{\mathrm{BB}}$ on the $\mathrm{HB}$ and $\mathrm{NB}$, suggesting a link between the emission from the neutron star and the electron temperature of
Rossi-XTE $\quad$ PCA + HEXTE

GX 5-1

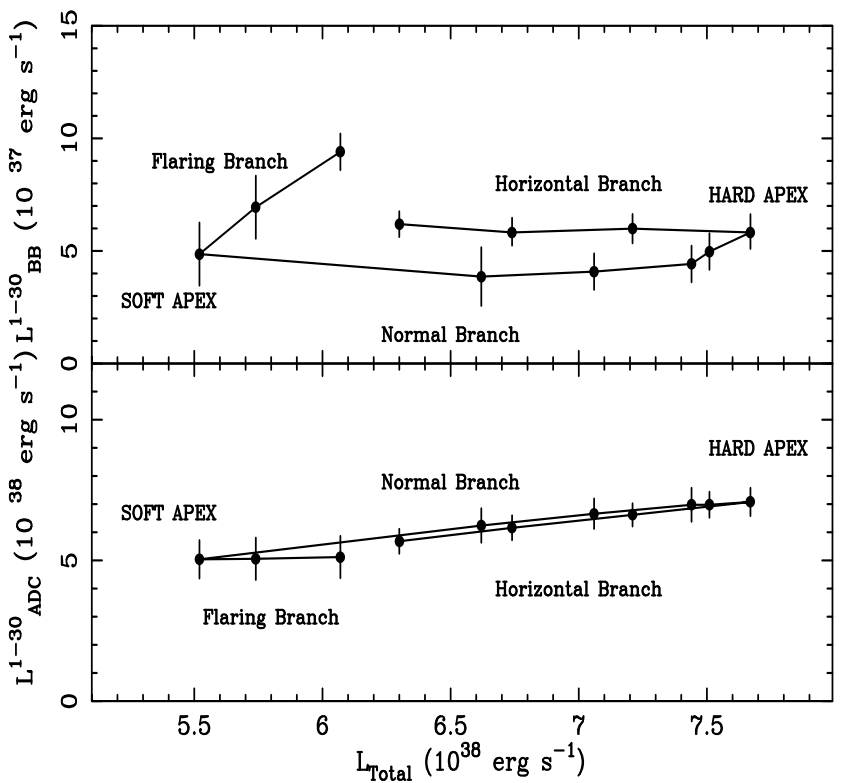

Fig. 5. Variation of emission component $1-30 \mathrm{keV}$ luminosities around the Z-track: upper panel: the blackbody luminosity; lower panel: the ADC Comptonized emission luminosity.

Rossi-XTE PCA + HEXTE

GX 5-1

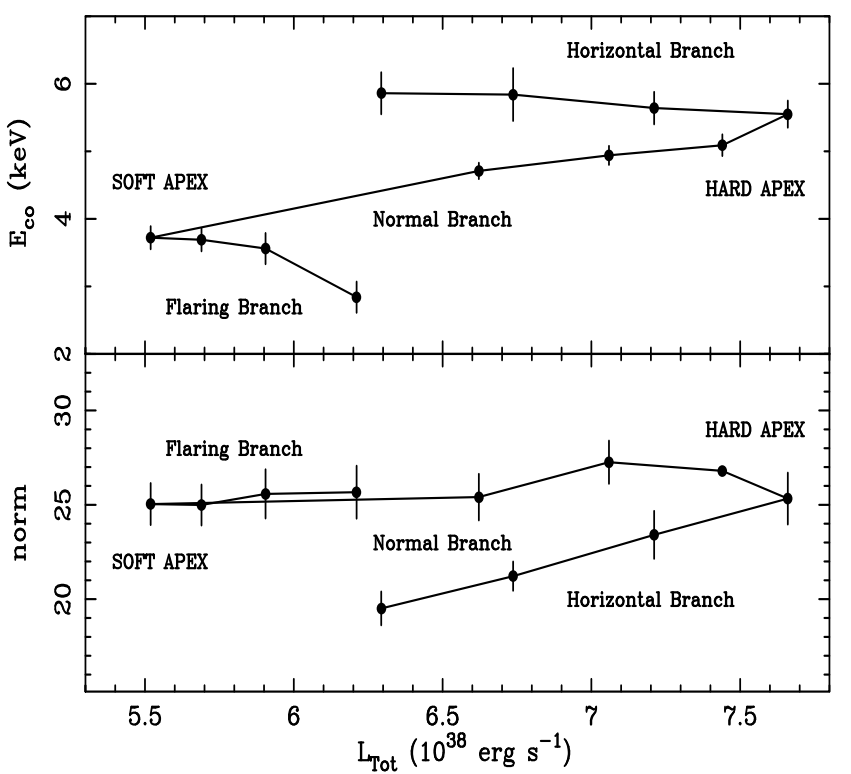

Fig. 6. The cut-off energy (upper panel) and normalization (lower panel) of the ADC Comptonized emission.

the ADC. For an ADC with high optical depth to electron scattering $E_{\mathrm{CO}}=3 k T_{\mathrm{e}}$ (Petrucci et al. 2001) whereas for low optical depth $E_{\mathrm{CO}}=k T_{\mathrm{e}}$. The present results allow a comparison of the neutron star blackbody temperature and $E_{\mathrm{CO}}$ and it is found that $E_{\mathrm{CO}} / k T_{\mathrm{BB}}$ has an average value of $2.7 \pm 0.5$ implying that the plasma is optically thick and that the ADC electron temperature is close to the neutron star blackbody temperature. The relationship of $k T_{\mathrm{BB}}$ and $k T_{\mathrm{e}}$ in LMXB was discussed by Bałucińska-Church \& Church (2005) who showed that in lower luminosity sources the ADC electron temperature $k T_{\mathrm{e}}$ was substantially higher than $k T_{\mathrm{BB}}$ so that there must be a presently-unknown heating process. However, in brighter sources $\left(L \gtrsim 2 \times 10^{37} \mathrm{erg} \mathrm{s}^{-1}\right)$, these two temperatures were 
found to be consistently equal, providing evidence for thermal equilibrium between the neutron star and corona.

\subsection{Timing analysis}

We next examine the results of carrying out timing analysis for the same selections of data along the Z-track as used in Sect. 3.1. Two types of data had been accumulated suitable for timing analysis up to $\mathrm{kHz}$ frequencies: event mode data of type E_125us_64M_24_1s and single bit data of types SB_125us_0_13_1s, SB_125us_14_17_1s and SB_125us_18_23_1s, i.e. one mode covering the energy band up to $20 \mathrm{keV}$ stored in three files. Power density spectra were extracted with $125 \mu$ s time resolution from both event mode and single bit data into a single power spectrum for each selection along the Z-track. The energy range was restricted to the band 5-60 keV for event mode data with the RXTE-specific ftool SEBITMASK specifying channels corresponding to these energies and applying this with FESELECT to the data files. Power spectra were then extracted using the Xronos facility POWSPEC which essentially rebinned all data into a single energy bin. A standard Leahy normalization was applied. In order to carry out high quality fitting of the power spectra, we converted the spectra to a format that allowed fitting with the Xspec spectral fitting software, requiring changes to the header of the fits files and re-naming of the columns to correspond to frequency, power and power error. Finally an output file was made suitable for use with Xspec using the ftool FLX2XSP producing a file with pha extension as for an energy spectrum. This ftool also created a dummy instrument response function as needed by Xspec consisting of a unitary matrix having no effect on the data. Deadtime correction was carried out and the spectra grouped to a minimum of 60 counts per bin as found suitable by trial and error.

The power spectra were fitted within the range 150-950 Hz, using a three-component model consisting of two Lorenzian lines for the kilohertz QPO plus a power law which was able to fit the continuum of the spectrum well within the restricted frequency range. As expected, $\mathrm{kHz} \mathrm{QPO}$ were only detected on the horizontal branch of the Z-track and the upper part of the normal branch, that is, from a total of 5 of the 11 positions at which spectral fitting was carried out. Figure 7 (lower panel) shows the best-fit frequencies obtained for the two QPO peaks in these spectra as a function of total luminosity $\left(L_{\mathrm{Tot}}\right)$ in the band $1-30 \mathrm{keV}$ obtained previously during spectral fitting. It can be seen that there is a monotonic systematic increase of the QPO frequency from the end of the HB to the point on the upper NB for both the upper and lower frequency QPO. Thus we find the same general trend of the $\mathrm{kHz}$ QPO frequencies as reported previously for GX 5-1 (Jonker et al. 2002) and in other Z-track sources (e.g. Jonker et al. 2000). The frequency difference $v_{2}-v_{1}$ is also shown in the lower panel and can be seen to have a minimum of $\sim 280 \mathrm{~Hz}$ at the hard apex and a maximum of $\sim 380 \mathrm{~Hz}$ part way along the horizontal branch. In terms of the $90 \%$ confidence uncertainties in $v_{2}$ and $v_{1}$ obtained in the fitting, typically $10-30 \mathrm{~Hz}$, the variation is significant. The ratio of frequencies $v_{2} / v_{1}$ is also shown in the upper panel. Although the ratio passes through the value 1.5 , i.e. the ratio $3: 2$ significant in the resonance model of QPO (Sect. 1), and interestingly this occurs at the hard apex, the ratio varies substantially around the Z-track from 1.4 on the normal branch to 2.4 at the end of the horizontal branch, only equalling 1.5 in one part of the track.

We next assume that the upper frequency $v_{2}$ corresponds to an orbital frequency in the inner disk and calculate the orbital
Rossi-XTE PCA

GX 5-1

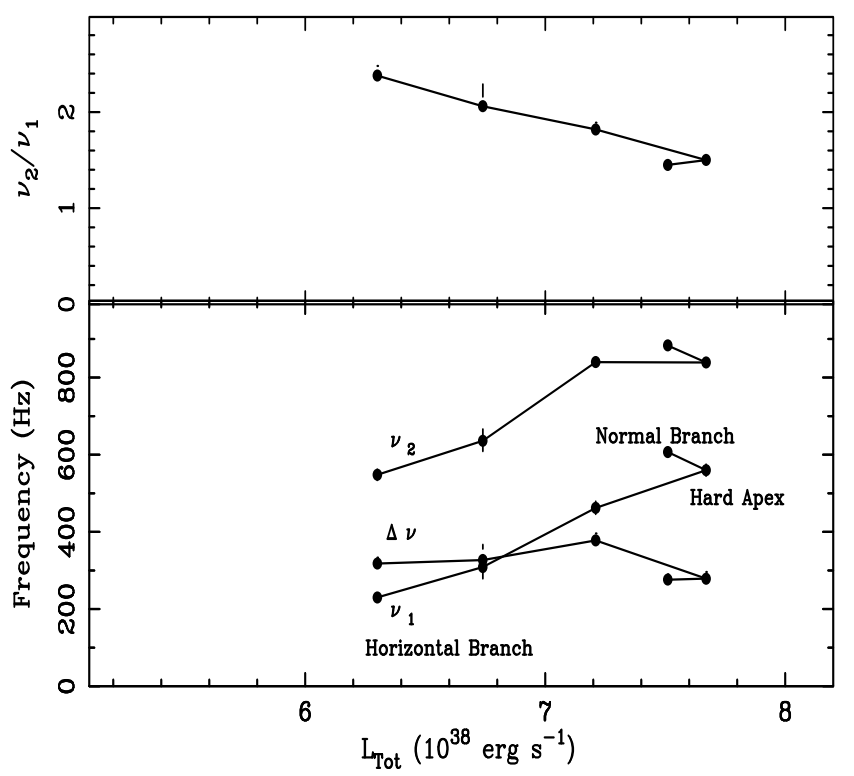

Fig. 7. Lower panel: the variation of lower frequency and upper frequency $\mathrm{kHz}$ QPO detected on the upper normal and horizontal branches as a function of the total $1-30 \mathrm{keV}$ luminosity $\left(L_{\mathrm{Tot}}\right)$ together with the difference $\Delta v$; top panel: variation of the frequency ratio $v_{2} / v_{1}$ around the Z-track.

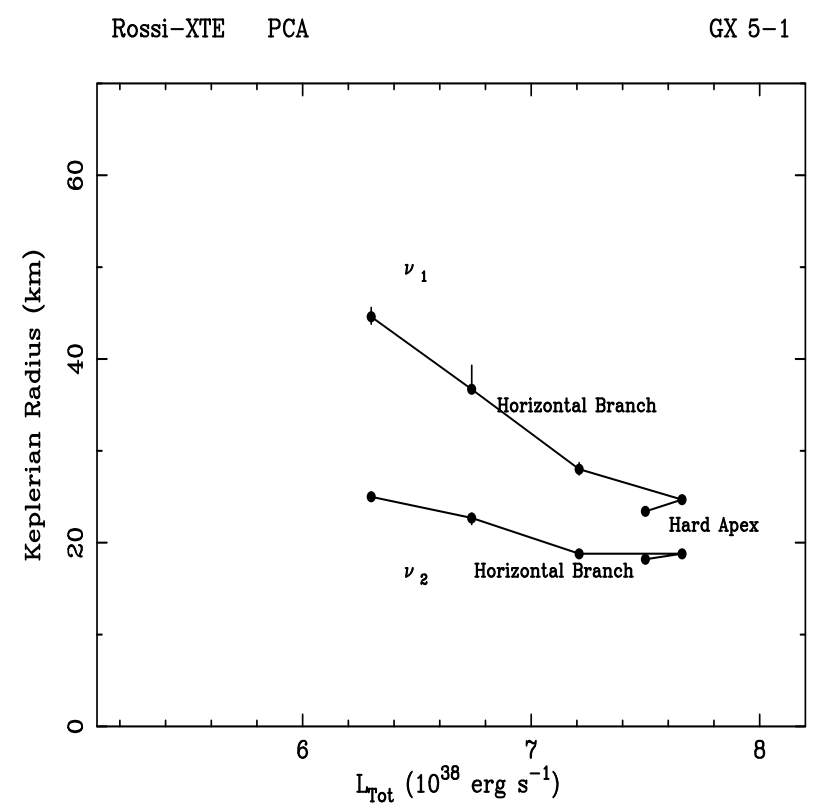

Fig. 8. The orbital radii of the upper and lower frequency QPO as a function of luminosity.

radii for both $\mathrm{kHz}$ QPO assuming Newtonian dynamics in which the orbital frequency at a radius $r$ in the disk is given by

$2 \pi v=\sqrt{\frac{G M}{r^{3}}}$,

where $M$ is the mass of the neutron star assumed to be $1.4 M_{\odot}$. These radial positions are shown in Fig. 8. For the higher frequency QPO, the frequency $v_{2}$ for the power spectrum in the normal branch corresponds to a radial position of about $18 \mathrm{~km}$, i.e. some distance away from the neutron star surface. 
Rossi-XTE $\quad$ PCA + HEXTE

GX 5-1

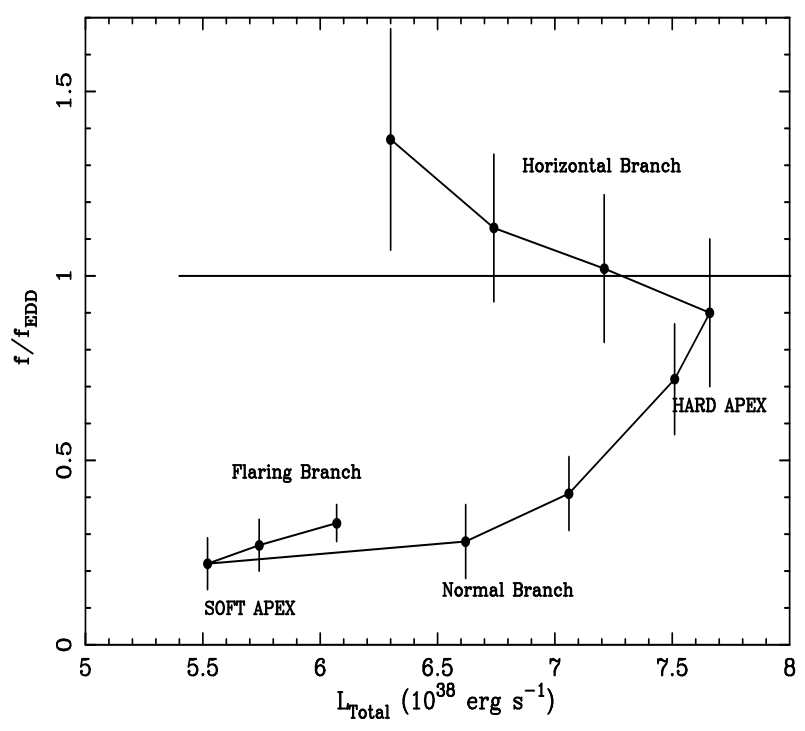

Fig. 9. The ratio of neutron star emitted flux to the Eddington flux as a function of position on the Z-track.

As the source moves on to the horizontal branch, the frequency decrease corresponds to a radial position moving outwards eventually reaching $25 \mathrm{~km}$ clearly suggesting that the source of oscillation moves further away from the neutron star. In terms of the spectral evolution along the Z-track, this is very suggestive, since both in the present work (Sect. 4.1; Fig. 9) and in our previous work on GX $340+0$ (Church et al. 2006), the radiation pressure at the inner disk becomes very strong on the $\mathrm{HB}$ and is expected to disrupt the inner disk removing the inner portions. Discussion of this will be postponed until after more detailed consideration of the spectral fitting results.

\section{Discussion}

\subsection{Spectral fitting results}

The results obtained in Sect. 3.1 show that the spectra of GX 5-1 are well-fitted by the extended ADC model, and that the evolution of spectral parameters around the Z-track suggest a relatively simple explanation of the Z-track as discussed below. The increase of ADC luminosity between the soft apex and hard apex provides evidence that $\dot{M}$ increases in this direction. The constancy of the ADC luminosity on the flaring branch combined with an increasing $L_{\mathrm{BB}}$ shows that flaring must consist of unstable nuclear burning on the neutron star. Both of these conclusions disagree with the often-held view that $\dot{M}$ increases monotonically in the direction $\mathrm{HB}-\mathrm{NB}-\mathrm{FB}$. Moreover, the spectral evolution of GX 5-1 around the Z-track is essentially the same as in the similar source GX $340+0$ (Church et al. 2006) so that the same explanation works for both sources. We discuss below effects taking place on the normal and horizontal branches and on the flaring branch in more detail.

\subsection{The effects of radiation pressure}

Moving away from the soft apex where the neutron star blackbody temperature is lowest and the blackbody radius indicates that the whole star is emitting, we find a substantial increase in $k T_{\mathrm{BB}}$, so that there is a large increase in the radiation pressure $\sim T^{4}$.
At the same time, the blackbody radius $R_{\mathrm{BB}}$ decreases to a value of $\sim 5 \mathrm{~km}$ at the $\mathrm{HB}$ end of the $\mathrm{Z}$-track so that the emission is from a reduced area on the neutron star, presumably an equatorial belt. It would be normal to describe the source in terms of its luminosity compared with the Eddington luminosity which is, of course, derived on the assumption of radial and not disk accretion and assuming accretion over $4 \pi$ steradians. In the present case, where only parts of the neutron star emit, it is instructive to compare the flux $f$ emitted by unit emitting area on the neutron star with the Eddington flux $L_{\mathrm{Edd}} / 4 \pi R^{2}$, where $R$ is the neutron star radius assumed to be $10 \mathrm{~km}$, so that $f_{\text {Edd }}=1.4 \times 10^{25} \mathrm{erg} \mathrm{cm}^{-2} \mathrm{~s}^{-1}$. When only parts of the neutron star are emitting, the flux $f$ will exceed the mean X-ray flux deduced from the measured luminosity and the source distance. Figure 9 shows the ratio of $f$ to $f_{\text {Edd }}$. The ratio has its lowest value at the soft apex of $\sim 0.2$ showing that radiation pressure is weak, rising to unity at the hard apex and continuing to rise to the end of the Z-track. Thus the radiation pressure of the emitting band becomes strong at the hard apex and horizontal branch such that the effects on the accretion flow will be strong. At the Eddington flux, the radiation pressure force balances the inwards gravitational force, both flux and gravitational force decreasing as $1 / r^{2}$ as in the standard derivation of the Eddington limit, except that this does not apply over the whole neutron star surface and the effects of radiation pressure will depend on which direction is considered.

Especially in luminous LMXB, the inner accretion disk is radiatively supported by the radiation pressure of the disk itself having an equilibrium half-height $H_{\mathrm{eq}}=3 \sigma_{\mathrm{T}} \dot{M} / 8 \pi m_{\mathrm{p}} c$, where $\sigma_{\mathrm{T}}$ is the Thomson cross section, $m_{\mathrm{p}}$ is the proton mass and $c$ the velocity of light (Frank et al. 2002). The height of the disk rises rapidly from zero to $H_{\text {eq }}$ in a radial distance $\sim 10 \mathrm{~km}$ from the surface of the neutron star, and for the hard apex in the present data, $H_{\text {eq }}$ is $65 \mathrm{~km}$ where $\dot{M}$ is derived from the total $1-30 \mathrm{keV}$ luminosity. Thus the inner disk rises high above the neutron star, and we propose that the strong radiation pressure at the hard apex and on the HB severely disrupts the inner disk blowing large parts of it away. At any radial and vertical position in the unperturbed inner disk $(r, z)$, the vertical force due to radiation pressure plus gas pressure is balanced by the vertically downwards component of the gravitational force. Radiation pressure from the neutron star acts on a line from the star to this point $(r$, $z$ ) offsetting the gravitational force. If this offset reduces the effective gravitational force close to zero, the equilibrium height of the disk would tend to infinity. Moreover, if we assume that $\dot{M}$ is maximum on this part of the Z-track, the radiation pressure of the disk itself will also be increased moving the disk surface upwards.

Clearly the combined effects of the radiation pressure of the neutron star and that of the inner accretion disk will be complicated and modelling should be carried out. That is beyond the scope of the present work, but we show in Fig. 10 an approximate schematic view of the expected effect. The unperturbed profile of the disk is shown as a dashed line, and the actual profile of the inner disk by a full line. The disk height $H(r)$ will revert to the unperturbed form of the radiatively-supported disk at some radial position $r_{\text {un }}$, and this position represents an effective inner edge of the disk, since at smaller radial positions there may be a residual disk, but of substantially reduced height.

The increase of column density found in spectral fitting on the normal branch supports the release of mass within the system on this part of the Z-track. Our measured increase of absorption indicates the absorber is spatially extended so that both emission components will be affected. It is clear that most of this 


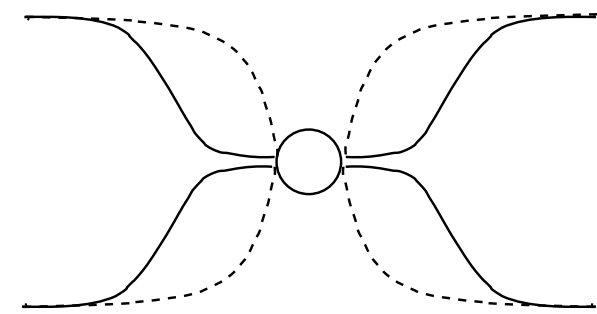

Fig. 10. Schematic view of the inner, radiatively supported inner accretion disk disrupted by radiation pressure; the unperturbed disk is shown as a dashed line. The radial profile of height $H(r)$ meets the unperturbed profile at radial position $r_{\text {un }}$ and it is proposed in the present work that the higher frequency $\mathrm{kHz}$ QPO consists of an oscillation taking place in the neighbourhood of $r_{\mathrm{un}}$.

material would not be fully ionized since the ionization parameter $\xi=L / n r^{2}$ decreases rapidly with distance and a $\xi$ of $10^{6}$ is required for there to be complete ionization (e.g. Makishima 1986). Moreover there would be a spatial variation of $\xi$ so that material located at vertical positions not directly facing the emitting part of the neutron star would be less ionized.

The localized radiation pressure of the neutron star as measured by $f / f_{\text {Edd }}$ rises by almost an order of magnitude from the soft apex to the end of the HB. Radio emission is non-existent on the FB and soft apex and strongest at the hard apex and HB. Thus in GX 5-1 as found previously in GX 340+0 there is a very good correlation between observation of radio and $f / f_{\text {Edd }}$ and we propose that strong radiation pressure is a necessary, but not necessarily sufficient condition for jet formation.

We also comment on the observed decrease of blackbody radius, i.e. emitting area on the neutron star in ascending the normal branch, noting that this is consistent with previous work. The blackbody radius obtained from spectral fitting $R_{\mathrm{BB}}$ is related to the half-height of the emitting equatorial strip on the neutron star $h$ by $4 \pi R_{\mathrm{BB}}^{2}=4 \pi R_{\mathrm{NS}} h$, where $R_{\mathrm{NS}}$ is the radius of the neutron star, assumed to be $10 \mathrm{~km}$. In a survey of LMXB with Asca and BeppoSAX, Church \& Bałucińska-Church (2001) made a survey comparing the blackbody luminosity $\left(L_{\mathrm{BB}}\right)$ in the survey sources with the Comptonized luminosity $\left(L_{\mathrm{ADC}}\right)$ in order to investigate the well-known discrepancy that $L_{\mathrm{BB}}$ in most LMXB is many times less than the fraction of the total luminosity expected $(\sim 50 \%)$ on the basis of simple theory. It was found that the half-height of the equatorial emitter on the neutron star $h$ was in the lower luminosity LMXB substantially less than the neutron star radius. However, in all sources $h$ followed the simple relation $h=H$, where $H$ is the half-height of the inner disk, valid over 3 decades of source luminosity. A probable explanation of this geometric equality is the model of accretion flow spreading on the neutron star (Inogamov \& Sunyaev 1999), which was shown to agree reasonably with the survey results (Church et al. 2002). Thus in lower luminosity sources, the spreading does not extend so far vertically, and the emitting region is smaller.

We have suggested that in the Z-track sources parts of the inner accretion disk are blown away by high radiation pressure presumably leaving a residual innermost disk of reduced height. Thus a reduced value of disk height $H$ is expected to lead to a reduced emitter size on the neutron star, and conversely, the observed decrease of $R_{\mathrm{BB}}$ and $h$ is evidence that the height of the inner disk $H$ is reduced from more than $50 \mathrm{~km}$ to $\sim 3 \mathrm{~km}$ indicating disruption of the inner disk.

\subsection{Flaring}

The constancy of $L_{\mathrm{ADC}}$ on the flaring branch and the increase of $L_{\mathrm{BB}}$ lead to the conclusion that flaring must be unstable nuclear burning on the neutron star, since if $\dot{M}$ increased as usually assumed we would definitely expect $L_{\mathrm{ADC}}$ to increase substantially as it does on the normal branch. Further support for this conclusion comes from comparing with the theory of unstable nuclear burning. Unstable burning is expected at various mass accretion rates (Fujimoto et al. 1981; Fushiki \& Lamb 1987; Bildsten 1998; Schatz et al. 1999), the various régimes of stable and unstable burning depending on the value of $\dot{m}$, the mass accretion rate per unit area of the neutron star. For $5.0 \times 10^{3}<\dot{m}<1.3 \times 10^{5} \mathrm{~g} \mathrm{~cm}^{-2} \mathrm{~s}^{-1}$ there is unstable $\mathrm{He}$ burning in a mixed $\mathrm{H} / \mathrm{He}$ environment, while for $\dot{m}>\dot{m}_{\mathrm{ST}}=$ $1.3 \times 10^{5} \mathrm{~g} \mathrm{~cm}^{-2} \mathrm{~s}^{-1}$, burning is always stable (Bildsten 1998), this value having an estimated uncertainty of $30 \%$ (giving a range of about $0.9-1.7 \times 10^{5} \mathrm{~g} \mathrm{~cm}^{-2} \mathrm{~s}^{-1}$ ). In deriving values of $\dot{m}$, we use actual values of the emitting area given by the blackbody radius obtained in spectral fitting, i.e. $\dot{m}=\dot{M} /\left(4 \pi R_{\mathrm{BB}}^{2}\right)$. At the soft apex, we find $\dot{m}=1.8 \pm 0.2 \times 10^{5} \mathrm{~g} \mathrm{~cm}^{-2} \mathrm{~s}^{-1}$ consistent with the critical value $\dot{m}_{\mathrm{ST}}$, and so it is expected theoretically that burning is stable on the normal branch but unstable on the flaring branch, the soft apex being the point at which the transition between these occurs.

If we progress along the FB away from the soft apex, the value of $\dot{m}$ derived from spectral fitting decreases, so that once started, unstable burning remains unstable. Moving in the opposite direction away from the soft apex, however, $\dot{m}$ rises substantially, to $3.6 \pm 0.5 \times 10^{5} \mathrm{~g} \mathrm{~cm}^{-2} \mathrm{~s}^{-1}$ for the first spectrum on the $\mathrm{NB}$, increasing eventually to $10 \times 10^{5} \mathrm{~g} \mathrm{~cm}^{-2} \mathrm{~s}^{-1}$ at the end of the HB. This is due to the decrease of blackbody emitting area and the increase of $\dot{M}$ and shows that conditions on the neutron star are well into the régime of stable burning.

It would be desirable to find other observational tests of whether flaring consists of nuclear burning and it would be interesting to derive an $\alpha$-parameter for flaring in GX 5-1, since the agreement of measured $\alpha$ values with theory was the essential proof that bursting was nuclear in nature. $\alpha$ is defined as the ratio of accretion luminosity to burst luminosity evaluated in bursting by integrating the non-burst luminosity between bursts, and the luminosity within a single burst. For a single burst, the time needed for the accretion flow to accumulate and to settle on the neutron star increasing in density towards ignition conditions is well-defined as the time between bursts. Theoretical values are obtained by dividing the accretion luminosity per nucleon $\left(G M / R_{\mathrm{NS}}\right.$ by the nuclear energy release per nucleon from which $\alpha \sim 30$ for H-burning and more than 100 for He burning. However, a detailed investigation of flaring in the Z-track sources in the context of nuclear burning has not been made and the physics of the accumulation of mass and settling on the star has not been investigated and it is unclear what timescale is involved in the matter involved coming to ignition conditions. Thus consideration of the $\alpha$-parameter is beyond the scope of the present work.

\subsection{The $\mathrm{kHz} Q P O$}

We are now able to discuss the QPO results in terms of the spectral fitting results, and we firstly take the QPO variation along the Z-track shown in Fig. 8 as the Keplerian radius of the oscillation versus $L_{\text {Tot }}$ and re-draw this as Keplerian radius as a function of $f / f_{\text {Edd }}$ as shown in Fig. 11 . 
Rossi-XTE PCA

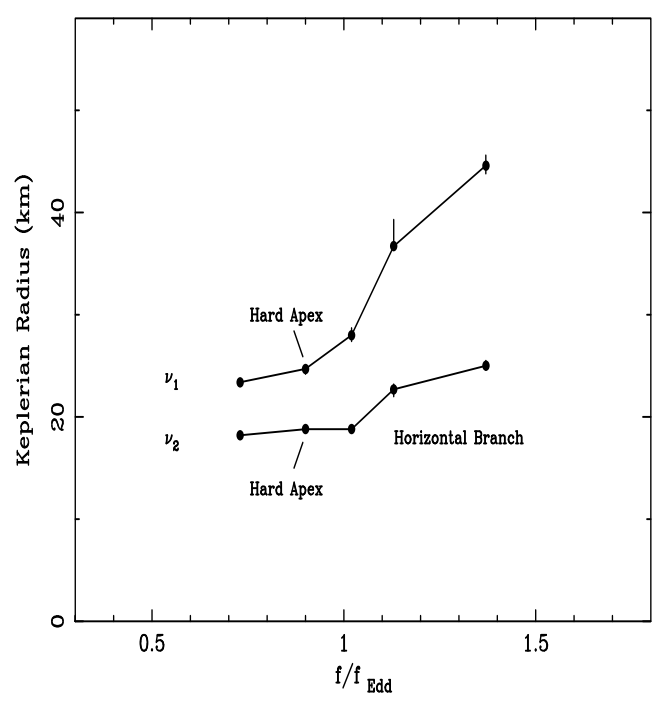

Fig. 11. Variation of Keplerian radius of the $\mathrm{kHz}$ QPOs as a function of radiation pressure of the neutron star, i.e. the emitted flux of the surface in units of the Eddington flux $f_{\text {Edd }}$ demonstrating a striking correlation between the QPO radial position of origin and high radiation pressure.

A striking feature can be seen that the major change in Keplerian radius and thus in QPO frequency takes place when $f / f_{\text {Edd }}$ equals unity. This result is unlikely to be coincidental and suggests that the strong radiation pressure causes the variation in QPO frequency along the Z-track. Moving from the hard apex to the end of the Z-track, $f / f_{\text {Edd }}$ increases from 1.0 to 1.5 and the Keplerian radius of the higher frequency QPO increases as the frequency decreases. The most obvious explanation is that this radiation pressure is indeed disrupting the inner disk so that the oscillations comprising the higher frequency QPO move outwards but staying on the inner edge or close to it, and this can explain the change of frequency. It is clear that the lower frequency QPO responds to the change in $v_{2}$ in some way but the results do not provide a clear indication of the mechanism of this. However, the result shown in Fig. 11 does suggest an alternative to existing ideas regarding possible truncation of the inner disk. Much theoretical work suggests that the accretion disk is in contact with the neutron star, e.g. the Inogamov \& Sunyaev model (1999) of accretion flow spreading on the surface of the neutron star, while other work considers the possibility that the disk is truncated at a specific radius, either at an innermost stable orbit, or at an Alfvén radius assumed external to the neutron star by capture onto the weak magnetic field of the neutron star or at a sonic point. In the sonic point model (Miller et al. 1998), part of the accretion flow can remain in the disk until it impacts on the neutron star. The present work suggests that the disk may be truncated, but only in part of the Z-track, and that the mechanism may be radiation pressure.

\section{Conclusions}

We have shown that spectra selected along the Z-track in GX 5-1 are well fitted using the extended ADC model, and that the spectral evolution is very similar to that we found in GX 340+0 (Church et al. 2006). Thus the physical model proposed to explain the spectral evolution in that source is also able to explain GX 5-1. In this model, the soft apex has the lowest value of $\dot{M}$ and the strong physical changes on the normal branch are primarily due to an increase of $\dot{M}$ and of neutron star temperature.
The increase of temperature means that the radiation pressure must be high in the neighbourhood of the neutron star. We propose that this super-Eddington radiation pressure combined with inner disk radiation pressure disrupts the inner disk diverting accretion flow into the vertical direction leading to jet formation. Thus high radiation pressure is a necessary but not necessarily sufficient condition for jet formation. On the horizontal branch, the decrease of intensity and $L_{\mathrm{ADC}}$ indicates that $\dot{M}$ decreases; however the blackbody temperature continues to increase and there are various ways of explaining this, such as $\dot{M}$ decreasing in the disk while an enhanced accretion flow is still affecting the neutron star. Evidence is presented that the flaring branch consists of unstable nuclear burning on the neutron star given the constant $L_{\mathrm{ADC}}$ and the increase of $L_{\mathrm{BB}}$, and the good agreement between $\dot{m}$ at the soft apex and the theoretical $\dot{m}_{\mathrm{ST}}$ below which burning is unstable. However, we should add the caution that while we now have evidence that the flaring branch can be explained in this way in the three Cyg-like Z-track sources, i.e. in GX 340+0 (Church et al. 2006), in GX 5-1 (present paper) and in Cyg X-2 (Church et al. 2007), we do not claim that this is so in the other group of Z-track sources: the Sco-like sources. Preliminary evidence suggests that these are similar on the NB and HB, but that the FB may be different.

Radio emission is detected at the hard apex and horizontal branch showing that jets are present (Penninx 1989; Hjellming \& Han 1995; Berendsen et al. 2000) where we find that radiation pressure is strongest and is non-existent on the FB and soft apex. It was, of course, previously suggested that radiation pressure may be important in jet formation (Bisnovatyi-Kogan \& Blinnikov 1977; Begelman \& Rees 1984) and that the conical opening in the inner accretion disk may perform some degree of collimation (Lynden-Bell 1978).

The present results do not support the standard view that $\dot{M}$ increases monotonically in the direction $\mathrm{HB}-\mathrm{NB}-\mathrm{FB}$, but have $\dot{M}$ constant in flaring and increasing from the soft apex to hard apex. Moreover, in the standard view the source becomes superEddington at the soft apex whereas the present work indicates the opposite, that this happens at the hard apex.

By combining timing and spectral analysis we have shown that the variation of the $\mathrm{kHz}$ QPO along the Z-track is highly correlated with $f / f_{\text {Edd }}$, and the major change in the QPO frequencies takes place when this ratio equals unity, and the radiation pressure becomes large. This suggests that the higher frequency $\mathrm{kHz} \mathrm{QPO}$ is an oscillation at the inner edge of the disk, i.e. the edge formed by the action of radiation pressure removing a large section of the unperturbed disk, and that the edge moves to larger radial positions when the radiation pressure increases. A major feature of $\mathrm{kHz}$ QPO is that they are essentially a feature of the horizontal branch, fading away at the upper end of the normal branch and this is consistent with our model for the Z-track as the high radiation pressure of the neutron star revealed by spectral fitting is also a feature of the horizontal branch. Figure 11 shows that when $f / f_{\text {Edd }}$ has fallen to $\sim 0.5, \mathrm{kHz}$ QPO are no longer seen. Thus there is an indication that the $\mathrm{kHz}$ QPO only exist when part of the inner disk is blown away. The smallest Keplerian radius we measure has a value of $18 \mathrm{~km}$ on the upper normal branch and if QPO continued to exist on the normal branch, the Keplerian radius would decrease further. However, as the radiation pressure falls there would be no truncation of the disk at an edge acting as a site for the oscillation, and this is consistent with the lack of $\mathrm{kHz}$ QPO on the lower normal branch and flaring branch.

Thus there is a simple explanation for the known occurrence of the higher frequency $\mathrm{kHz}$ QPO at a preferred radial position 
in the disk: that this is the disk edge, and the results suggest that the variation of frequency is caused by change in position of the edge.

The physics of the accretion flow in the inner disk is very uncertain (e.g. van der Klis 2000), and probably the most important question is whether the disk in general in LMXB meets the neutron star or is truncated at an innermost stable orbit, an Alfvén radius or a sonic point, and this is relevant to the QPO models which invoke truncation. However, it is difficult to say whether truncation of the disk due to relativistic, magnetospheric or other effects actually takes place. The present work presents a simple observational result on the higher frequency QPO and does not directly say anything about the lower frequency oscillation, and it is not appropriate to comment on the detailed physics of the QPO models here. However, the present work indicates that the inner disk is not in general truncated, but can become truncated on the upper NB and HB. Disk truncation is due to high radiation pressure and we do not need to invoke truncation because of an innermost stable orbit, or an Alfvén radius external to the neutron star or a sonic point.

Acknowledgements. This work was supported in part by the Polish KBN grant KBN-1528/P03/2003/25, by the Polish Ministry of Higher Education and Science grant no. 3946/B/H03/2008/34 and by PPARC grant PPA/G/S/2001/00052.

\section{References}

Abramowicz, M. A., \& Kluźniak, W. 2001, A\&A, 374, L19

Abramowicz, M. A., Bulik, T., Bursa, M., \& Kluźniak, W. 2003, A\&A, 404, L21

Agrawal, V. K., \& Sreekumar, P. 2003, MNRAS, 346, 933

Asai, K., Dotani, T., Mitsuda, K., et al. 1994, PASJ, 46, 479

Asai, K., Dotani, T., Nagase, F., \& Mitsuda, K. 2000, ApJS, 131, 571

Bałucińska-Church, M., \& Church, M. J. 2005, Proc of Interacting Binaries: Accretion, Evolution and Outcomes, Cefalu, July 2004, AIP Conf. Proc., New York, 797, 339

Bałucińska-Church, M., Church, M. J., Oosterbroek, T., et al. 1999, A\&A, 349, 495

Bałucińska-Church, M., Humphrey, P. J., Church, M. J., \& Parmar, A. N. 2000, A\&A, 360, 583

Bałucińska-Church, M., Barnard, R., Church, M. J., \& Smale, A. P. 2001, A\&A, 378,847

Barnard, R., Bałucińska-Church, M., Smale, A. P., \& Church, M. J. 2001, A\&A, 380, 494

Barnard, R., Church, M. J., \& Bałucińska-Church, M. 2003, A\&A, 405, 237

Begelman, M. C., \& Rees, M. J. 1984, MNRAS, 206, 209

Belloni, T., Méndez, M., \& Homan, J. 2005, A\&A, 437, 209

Berendsen, S. G. H., Fender, R., Kuulkers, E., Heise, J., \& van der Klis, M. 2000, MNRAS, 318, 599

Bildsten, L. 1998, in Proc NATO ASIC 515, The Many Faces of Neutron Stars, ed. R. Buccheri, J. van Paradijs, \& M. A. Alpar (Dordrecht: Kluwer), 419

Bildsten, L., Proc of Cosmic Explosions: Tenth Astrophysics Conf., ed. S. S. Holt, \& W. H. Zhang, AIPC, 522, 359

Bisnovatyi-Kogan, G. S., \& Blinnikov, S. I. 1977, A\&A, 59, 111

Bradt, H., Naranan, S., Rappaport, S., \& Spada, G. 1968, ApJ, 152, 1005

Casella, P., Belloni, T., \& Stella L. 2006, A\&A, 446, 579

Church, M. J., \& Bałucińska-Church, M. 1995, A\&A, 300, 441

Church, M. J., \& Bałucińska-Church, M. 2001, A\&A, 369, 915

Church, M. J., \& Bałucińska-Church, M. 2004, MNRAS, 348, 955

Church, M. J., Mitsuda, K., Dotani, T., et al. 1997, ApJ, 491, 388

Church, M. J., Bałucińska-Church, M., Dotani, T., \& Asai, K. 1998a, ApJ, 504, 516

Church, M. J., Parmar, A. N., Bałucińska-Church, M., et al. 1998b, A\&A, 338, 556

Church, M. J., Inogamov, N. A., \& Bałucińska-Church, M. 2002, A\&A, 390, 139 Church, M. J., Reed, D., Dotani, T., Bałucińska-Church, M., \& Smale, A. P. 2005, 359, 1336

Church, M. J., Halai, G. S., \& Bałucińska-Church, M. 2006, A\&A, 460, 233

Church, M. J., Bałucińska-Church, M., Jackson, N. K., \& Chin, J. 2007, A\&A, 8,191
Christian, D. J., \& Swank, J. H. 1997, ApJS, 109, 177

d'Ai, A., Zycki, P., di Salvo, T., et al. 2007, ApJ, 667, 411

Dickey, J. M., \& Lockman, F. J. 1990, Ann. Rev. Astron. Astrofis. 28, 215

di Salvo, T., Stella, L., Robba, N. R., et al. 2000, ApJ, 544, L119

di Salvo, T., Robba, N. R., Iaria, R., et al. 2001, ApJ, 554, 49

di Salvo, T., Farinelli, R., Burderi, L., et al. 2002, A\&A, 386, 535

Dieters, S., \& van der Klis, M. 2000, MNRAS, 311, 201

Done, C., Zycki, P., \& Smith, D. A. 2002, MNRAS, 331, 453

Fisher, P. C., Jordan, W. C., Meyerott, A. J., Acton, L. W., \& Roethig, D. T. 1968, ApJ, 151, 1

Fomalont, E. B., Geldzahler, B. J., \& Bradshaw, C. F. 2001, ApJ, 558, 283

Frank, J., King, A. R., \& Raine, D. J. 2002, Accretion power in astrophysics, third edn. (Cambridge: University Press)

Fujimoto, M. Y., Hanawa, T., \& Miyaji, S. 1981, ApJ, 247, 267

Fushiki, I., \& Lamb, D. Q. 1987, ApJ, 323, L55

Hasinger, G. 1988, Proc. Symp. Physics of Neutron Stars and Black Holes (Tokyo: Universal Academic Press), 97

Hasinger, G., \& van der Klis, M. 1989, A\&A, 225, 79

Hasinger, G., van der Klis, M., Ebisawa, K., Dotani, T., \& Mitsuda, K. 1990, A\&A, 235, 131

Hjellming, R. M., \& Han, X. 1995, X-Ray Binaries ed. W. H. G. Lewin, J. van Paradijs, \& E. P. J. van den Heuvel (Cambridge and New York: Cambridge University Press), 308

Homan, J., van der Klis, M., Jonker, P. G., et al. 2002, ApJ, 568, 878

Inogamov, N. A., \& Sunyaev, R. A. 1999, AstL, 25, 269

Jahoda, K., Swank, J. H., Giles, A. B., et al. 1996, SPIE, 2808, 59

Jonker, P. G., van der Klis, M., Wijnands, R., et al. 2000, ApJ, 537, 374

Jonker, P. G., van der Klis, M., Homan, J., et al. 2002, MNRAS, 333, 665

Kluźniak, W., \& Abramowicz, M. A., Bursa, M., \& Török, G. 2007, Rev. Mex. Astron. Astrofis. 27, 18

Kuulkers, E., van der Klis, M., Oosterbroek, T., et al. 1994, A\&A, 289, 759

Kuulkers, E., Homan, J., van der Klis, M., Lewin, W. H. G., \& Méndez, M. 2002, A\&A, 382, 947

Lamb, F. K. 1989 in Proc 23rd ESLAB Symp. on two Topics in X-ray Astronomy, ed. J. Hunt, \& B. Battrick, ESA SP-296, 1, 215

Lamb, F. K. 1991, in Neutron Stars: Theory and Observation, ed J. Ventura, \& D. Pines (Dordecht: Kluwer), 445

Lamb, F. K., \& Miller, M. C. 2003 [arXiv: astro-ph/0308179]

Lewin, W. H. G., Lubin, L. M., Tan, J., et al. 1992, MNRAS 256, 545

Lynden-Bell, D. 1978, Phys. Scrip., 17, 185

Makishima, K. 1986, The Physics of Accretion onto Compact Objects, Proc. of Tenerife workshop (Springer-Verlag) Lect. Notes Phys., 266, 249

Miller, G. S. 1990, ApJ, 356, 572

Miller, M. C., \& Lamb, F. K. 1993, ApJ, 413, L43

Miller, M. C., \& Lamb, F. K. 1996, ApJ, 470, 1033

Miller, M. C., Lamb, F. K., \& Psaltis, D. 1998, ApJ, 598, 791

Mitsuda, K., Inoue, H., Nakamura, N., \& Tanaka, Y. 1989, PASJ, 41, 97

Penninx, W. 1989, in Proc. of the 23rd ESLAB symposium on Two Topics in

X-ray Astronomy, ed. J. Hunt, \& B. Battrick, Bologna, ESA SP-296, 185

Petrucci, P. O., Haardt, F., Maraschi, L., et al. 2001, ApJ, 556, 716

Priedhorsky, W., Hasinger, G., Lewin, W. H. G., et al. 1986, ApJ, 306, L91

Psaltis, D., Lamb, F. K., \& Miller, G. S. 1995, ApJ, 454, L137

Schatz, H., Bildsten, L., Cumming, A., \& Wiescher, M. 1999, ApJ, 524, 1014

Schulz, N. S., \& Wijers, R. A. M. J. 1993, A\&A, 273, 123

Schulz, N. S., Hasinger, G., \& Trümper, J. 1989, A\&A, 225, 48

Shapiro, S. L., Lightman, A. P., \& Eardley, D. M. 1976, ApJ, 204, 187

Smale, A. P., Church, M. J., \& Bałucińska-Church, M. 2001, ApJ, 550, 962

Smale, A. P., Church, M. J., \& Bałucińska-Church, M. 2002, ApJ, 581, 1286

Stella, L., \& Vietri, M. 1998 ApJ, 492, L59

Strohmayer, T. 2001, ApJ, 552, L49

Sunyaev, R. A. 1973, SvA, 16, 941

van der Klis, M. 2000, ARA\&A, 38,717

van der Klis, M. 2006, in Compact Stellar X-ray Sources, ed. W. H. G. Lewin,

\& M. van der Klis (Cambridge: Cambridge University Press)

van der Klis, M., Jansen, F., van Paradijs, et al. 1985, Nature, 316, 225

van der Klis, M., Stella, L., White, N. E., Jansen, F., \& Parmar, A. N. 1987, ApJ, 316,411

van der Klis, M., Kitamoto, S., Tsunemi, H., \& Miyamoto, S. 1991, MNRAS, 248,751

van Paradijs, J., Penninx, W., \& Lewin, W. H. G. 1988, MNRAS, 233, 437

Vrtilek, S. D., Raymond, J. C., Garcia, M. R., et al. 1990, A\&A, 235, 162

Wijnands, R., van der Klis, M., Psaltis, D., et al. 1996, ApJ, 469, L5

Wijnands, R., Méndez, M., van der Klis, M., et al. 1998, ApJ, 504, L35

Wijnands, R., van der Klis, M., Homan, J., et al. 2003, Nature (Lett.), 424, 44 